\title{
Properties of Matrix Orthogonal Polynomials via their Riemann-Hilbert Characterization
}

\author{
F. Alberto GRÜNBAUM ${ }^{\dagger}$, Manuel D. DE LA IGLESIA $\ddagger$ \\ and Andrei MARTÍNEZ-FINKELSHTEIN ${ }^{\S}$ \\ $\dagger$ Department of Mathematics, University of California, Berkeley, Berkeley, CA 94720 USA \\ E-mail:grunbaum@math.berkeley.edu \\ URL: http://math.berkeley.edu/ grunbaum/ \\ ¥ Departamento de Análisis Matemático, Universidad de Sevilla, \\ Apdo (P. O. BOX) 1160, 41080 Sevilla, Spain \\ E-mail:mdi29@us.es \\ URL: http://euler.us.es/ mdi29/ \\ $\S$ Departamento de Estadística y Matemática Aplicada, Universidad de Almería, \\ 04120 Almería, Spain \\ E-mail: andrei@ual.es \\ URL: http://www.ual.es/ andrei/
}

Received June 09, 2011, in final form October 20, 2011; Published online October 25, 2011

http://dx.doi.org/10.3842/SIGMA.2011.098

\begin{abstract}
We give a Riemann-Hilbert approach to the theory of matrix orthogonal polynomials. We will focus on the algebraic aspects of the problem, obtaining difference and differential relations satisfied by the corresponding orthogonal polynomials. We will show that in the matrix case there is some extra freedom that allows us to obtain a family of ladder operators, some of them of 0 -th order, something that is not possible in the scalar case. The combination of the ladder operators will lead to a family of second-order differential equations satisfied by the orthogonal polynomials, some of them of 0 -th and first order, something also impossible in the scalar setting. This shows that the differential properties in the matrix case are much more complicated than in the scalar situation. We will study several examples given in the last years as well as others not considered so far.
\end{abstract}

Key words: matrix orthogonal polynomials; Riemann-Hilbert problems

2010 Mathematics Subject Classification: 42C05; 35Q15

\section{Introduction}

The theory of matrix orthogonal polynomials on the real line (MOPRL) has its foundations in the seminal papers of Krein [42, 43] (see also their account in the book of Berezans'kiu [2]). For further historical background and analytic results, the reader is referred to the survey [11] and to Chapter 4 of [51]. In many aspects the MOPRL resemble their scalar counterparts, especially where the proofs are based on basic properties of Hilbert spaces. Nevertheless, the non-commutativity of matrix multiplication and the existence of non-zero singular matrices add features to the theory that make MOPRL an interesting object of study. Moreover, many problems for scalar polynomials are better understood or recast in terms of some matrix polynomials, see, for instance, [26].

Recall that a matrix polynomial of degree $\leq n$ in $\mathbb{C}^{N \times N}$ and a scalar variable $x$ can be defined as an expression of the form

$$
\boldsymbol{A}_{n} x^{n}+\cdots+\boldsymbol{A}_{1} x+\boldsymbol{A}_{0},
$$


where $\boldsymbol{A}_{j}$ 's are constant matrices in $\mathbb{C}^{N \times N}$. In what follows we consider $N$ fixed, and denote by $\mathbb{P}_{n}$ the family of matrix polynomials of degree $\leq n$ in $\mathbb{C}^{N \times N}$, as well as by $\mathbb{P}:=\bigcup_{n \geq 0} \mathbb{P}_{n}$. We use preferably boldface letters to denote matrices, and standard font for scalars. We also use $\boldsymbol{I}_{N}$ for the $N \times N$ identity matrix, omitting the explicit reference to its dimension when it cannot lead anyone into confusion.

In this paper we consider some aspects of the MOPRL theory, assuming that the orthogonality is given by an absolutely continuous measure on the line. More precisely, our starting point is a weight $\boldsymbol{W}=\left(W_{i j}\right):(a, b) \rightarrow G L(N, \mathbb{R})$, defined and positive definite on a finite or infinite interval $(a, b) \subset \mathbb{R}$. We will assume that all $W_{i j}$ and $W_{i j}^{\prime}$ have finite moments:

$$
\int_{a}^{b}|x|^{n} \boldsymbol{W}(x) d x<\infty, \quad \int_{a}^{b}|x|^{n} \boldsymbol{W}^{\prime}(x) d x<\infty, \quad n \in \mathbb{N}_{0}:=\mathbb{N} \cup\{0\},
$$

where the integration of a matrix function is applied entry-wise.

For any two $\boldsymbol{P}, \boldsymbol{Q} \in \mathbb{P}$, the weight $\boldsymbol{W}$ induces two matrix-valued "inner products",

$$
(\boldsymbol{P}, \boldsymbol{Q})_{\boldsymbol{W}}=\int_{a}^{b} \boldsymbol{P}(x) \boldsymbol{W}(x) \boldsymbol{Q}^{*}(x) d x,
$$

and

$$
\langle\boldsymbol{P}, \boldsymbol{Q}\rangle_{\boldsymbol{W}}=\int_{a}^{b} \boldsymbol{Q}^{*}(x) \boldsymbol{W}(x) \boldsymbol{P}(x) d x=\left(\boldsymbol{Q}^{*}, \boldsymbol{P}^{*}\right)_{\boldsymbol{W}},
$$

where the asterisk denotes the conjugate transpose (or Hermitian conjugate) of a matrix. Due to this connection between both inner products, we restrict our attention to $(\cdot, \cdot)_{\boldsymbol{W}}$. We define also the norm

$$
\|\boldsymbol{P}\|_{W}:=\left(\operatorname{Tr}\langle\boldsymbol{P}, \boldsymbol{P}\rangle_{\boldsymbol{W}}\right)^{1 / 2},
$$

and assume that $\boldsymbol{W}$ is non-trivial, in the sense that $\|\boldsymbol{P}\|_{\boldsymbol{W}}>0$ for every non-zero matrix polynomial $\boldsymbol{P}$. In this case (see [11, Lemma 2.3] or [51, Proposition 4.2.3]), $(\boldsymbol{P}, \boldsymbol{P})_{\boldsymbol{W}}$ is nonsingular for every non-zero polynomial $\boldsymbol{P}$, and we can easily implement a matrix analogue of the Gram-Schmidt orthogonalization procedure, which yields a unique sequence $\left(\widehat{\boldsymbol{P}}_{n}\right)_{n}$ of monic orthogonal polynomials such that $\widehat{\boldsymbol{P}}_{0}=\boldsymbol{I}$,

$$
\widehat{\boldsymbol{P}}_{n}(x)=x^{n} \boldsymbol{I}+\sum_{j=0}^{n-1} \boldsymbol{a}_{n, j} x^{j}, \quad\left(\widehat{\boldsymbol{P}}_{n}, \boldsymbol{Q}\right)_{\boldsymbol{W}}=\mathbf{0} \quad \text { for every } \quad \boldsymbol{Q} \in \mathbb{P}_{n-1}, \quad n \in \mathbb{N},
$$

as well as matrix polynomials $\left(\boldsymbol{P}_{n}\right)_{n}$, "orthonormal" with respect to $\boldsymbol{W}$, such that

$$
\boldsymbol{P}_{n}(x)=\boldsymbol{\kappa}_{n} \widehat{\boldsymbol{P}}_{n}, \quad\left(\boldsymbol{P}_{n}, \boldsymbol{P}_{m}\right)_{\boldsymbol{W}}=\delta_{n, m} \boldsymbol{I} .
$$

Obviously, $\boldsymbol{P}_{n}$ 's are determined up to a unitary left factor, so we can speak about an equivalence class of orthonormal MOPRL, corresponding to the weight $\boldsymbol{W}$. Additionally, if $\boldsymbol{B}$ is a constant non-singular matrix, then

$$
\widetilde{\boldsymbol{W}}(x)=\boldsymbol{B} \boldsymbol{W}(x) \boldsymbol{B}^{*}
$$

is also a weight, and $\left(\widetilde{\boldsymbol{P}}_{n}\right)_{n}=\left(\boldsymbol{P}_{n} \boldsymbol{B}^{-1}\right)_{n}$ is the corresponding sequence of orthonormal polynomials. Hence, more than a single weight we consider an equivalence class of weights given by its representative $\boldsymbol{W}$. In particular, without loss of generality we can assume that $\boldsymbol{W}\left(x_{0}\right)=\boldsymbol{I}$ at a point $x_{0} \in(a, b)$. If this class contains a diagonal matrix-valued function, we say that $\boldsymbol{W}$ 
reduces to scalar weights. A characterization of this fact, as shown in [23], is the commutativity condition

$$
\boldsymbol{W}(x) \boldsymbol{W}(y)=\boldsymbol{W}(y) \boldsymbol{W}(x), \quad x, y \in[a, b] .
$$

This situation is considered trivial, and usually omitted from consideration.

Work in the last few years has revealed a number of explicit families of MOPRL; in many cases they are joint eigenfunctions of some fixed differential operator with matrix coefficients independent of the degree $n$ of the MOPRL. This study was initiated in [21], but nontrivial examples had to wait until $[23,29,34,36]$. A solution to the classification problem for those $\boldsymbol{W}$ whose MOPRL are common eigenfunctions of some fixed differential operator remains elusive. There are by now two methods that have yielded nontrivial examples: a) the connection with matrix-valued spherical functions for symmetric spaces [34, 36], and b) a combination of classical methods and some Lie algebra tools [23, 24]. A necessary condition in terms of moments is given in [23], and a necessary and sufficient condition in terms of the "ad-conditions" is given in [38]. In this approach one gets an explicit formula for one differential operator. Concerning applications, the recurrent relations have been used in the study of certain quasi-birth-and-death processes [30, 31, 32, 33], in which case the differential operator plays no role. Recently, new applications of these processes have been related to urn or Young diagrams models [37].

A big boost in the research of the scalar OPRL has been their Riemann-Hilbert (RH) characterization, introduced in the seminal paper of Fokas, Its and Kitaev [28], and complemented with a non-linear steepest descent analysis in a series of works of Deift, Zhou and collaborators $[12,13,16,17]$. This combination has allowed to establish extremely strong asymptotic results with applications in random matrix theory, in particular for unitary invariant ensembles [12, 14], determinantal point processes [8, 9, 44, 45], orthogonal Laurent polynomials [46, 47], Painlevé trascendents [10, 41], the Toda lattices [15], to mention a few.

The RH characterization, even without the steepest descent analysis, allows one to prove other algebraic and analytic properties of orthogonal polynomials, as it was illustrated in [13] for OP on the unit circle, and in [40, Chapter 22], for classical families of OPRL. One of the goals of this paper is to extend these considerations to MOPRL, something that, surprisingly enough, has not been explored in depth so far. We will show that the $\mathrm{RH}$ formulation, which has a very natural block-wise generalization to the MOPRL case, allows one to reveal some subtleties hidden in the matrix case. For instance, we will derive identities and difference-differential equations that were unknown even for some of the explicit families mentioned above. The RH technique can be also a natural method for obtaining the differential relations for MOPRL with respect to general weight matrices.

There have been several papers considering the block-wise $\mathrm{RH}$ problem of the flavor similar to the one discussed in this paper, but for singular (namely, rank 1) weight matrices. For these weights the MOPRL are connected with a class of multiple orthogonal polynomials that find applications in the analysis of determinantal point processes and non-intersecting stochastic paths (see e.g. the works of Kuijlaars and collaborators $[8,18,19,45]$ ) or of the multicomponent 2D Toda lattice hierarchy [1].

This paper deals with strictly algebraic consequences of the $\mathrm{RH}$ formulation of matrix polynomials orthogonal with respect to a weight matrix supported on an interval $[a, b] \subset \mathbb{R}$; the asymptotic analysis via the Deift-Zhou non-linear steepest descent method will not be discussed here.

In Section 2 we will discuss two dual RHPs, which are uniquely solved in terms of the MOPRL. Standard arguments related to RHP allow us to obtain the three-term recurrence relation and the differential identities (ladder operators) for MOPRL. Both yield also the so-called Lax pair for the polynomials, which is an over-determined system (2.28), whose compatibility conditions 
render further properties of MOPRL. All the results in this section have been previously obtained using different approaches, but the $\mathrm{RH}$ method will provide new and straightforward proofs.

In Section 3 we will consider a special transformation of the original RHP, based on factorizations of the weight matrix of the form $\boldsymbol{W}=\boldsymbol{T} \boldsymbol{T}^{*}$. The main goal is to obtain a RHP with constant jumps (independent of $z$ ), in order to simplify the differential relation obtained earlier. For simplicity, we will focus on the case where the support of the weight matrix is $\mathbb{R}$. The extension of the method to weights supported on semi-infinite or finite intervals is simple, see a short discussion at the end of this paper.

We exploit also the non-uniqueness of the weight factorization, which in the matrix case gives us some extra freedom and yields non-trivial relations. In particular, a family of ladder operators, some of them of 0 -th order, is obtained, a phenomenon that is not possible in the scalar situation. Furthermore, by combining appropriately the family of ladder operators, we will get the 0 -th, first and second order differential equations satisfied by the MOPRL. Some examples of lower order differential operators appear in $[5,6,35]$.

The considerations so far have been completely general, applicable to any family of MOPRL. In Section 4 we narrow the analysis of Sections 2 and 3 to some relevant examples of MOPRL supported on $\mathbb{R}$, obtaining new results even in the cases studied previously in the literature.

In the final stage of preparation of this manuscript we learned about the preprint [4] which also uses the Riemann-Hilbert approach for the analysis of the matrix orthogonal polynomial. Although there is some overlapping between the results contained in our Sections 2, 3 and in [4, Section 2], the focus of both contributions is different, and in this sense, complementary.

\section{The Riemann-Hilbert problem for MOPRL}

\subsection{Formulation and basic properties}

In this section we discuss the Riemann-Hilbert problem (RHP) related to MOPRL with respect to a $N \times N$ weight matrix $\boldsymbol{W}$ supported on an interval $[a, b] \subset \mathbb{R}$; this interval can be either bounded or unbounded. We assume $\boldsymbol{W}$ continuous and non-vanishing on $(a, b)$, and that at any finite endpoint of the support the weight $\boldsymbol{W}$ has at worse a power-type singularity, that is, $\boldsymbol{W}$ is of the form

$$
\boldsymbol{W}(z)=|z-c|^{\gamma_{c}} \widetilde{\boldsymbol{W}}(z), \quad \gamma_{c}>-1,
$$

where $c \in\{a, b\}, c \neq \pm \infty$, and $\widetilde{\boldsymbol{W}}$ is a bounded, continuous and non-vanishing at $z=c$. This class of weights comprises all the examples considered so far in the literature.

As a convention, in what follows we write the $2 N \times 2 N$ matrices partitioned into $N \times N$ blocks, as in (2.2) below. Recall that $\boldsymbol{A}^{*}$ stands for the Hermitian conjugate of the matrix $\boldsymbol{A}$, as well as $\boldsymbol{A}^{-*}=\left(\boldsymbol{A}^{*}\right)^{-1}$. We adopt the convention that for any matrix-valued function $\boldsymbol{P}(z)$ of a complex variable $z, \boldsymbol{P}^{*}$ denotes the matrix-valued function obtained as

$$
\boldsymbol{P}^{*}(z):=(\boldsymbol{P}(\bar{z}))^{*} .
$$

The RHP for MOPRL with respect to a weight matrix $\boldsymbol{W}$ consists in finding a matrix function $\boldsymbol{Y}^{n}: \mathbb{C} \rightarrow \mathbb{C}^{2 N \times 2 N}$ such that

(Y1) $\boldsymbol{Y}^{n}$ is analytic in $\mathbb{C} \backslash[a, b]$.

(Y2) $\boldsymbol{Y}^{n}$ has on $(a, b)$ continuous boundary values $\boldsymbol{Y}_{+}^{n}$ (resp., $\boldsymbol{Y}_{-}^{n}$ ) from the upper (resp., lower) half plane, such that

$$
\boldsymbol{Y}_{+}^{n}(x)=\boldsymbol{Y}_{-}^{n}(x)\left(\begin{array}{c:c}
\boldsymbol{I}_{N} & \boldsymbol{W}(x) \\
\hdashline \mathbf{0} & \boldsymbol{I}_{N}
\end{array}\right), \quad x \in(a, b) .
$$


(Y3) As $z \rightarrow \infty$, for every $m \in \mathbb{N}$ we have

$$
\boldsymbol{Y}^{n}(z)=\left(\boldsymbol{I}_{2 N}+\sum_{i=1}^{m} \frac{\boldsymbol{Y}_{i}^{n}}{z^{i}}+\mathcal{O}\left(1 / z^{m+1}\right)\right)\left(\begin{array}{c:c}
z^{n} \boldsymbol{I}_{N} & \mathbf{0} \\
\hdashline \mathbf{0} & z^{-n} \boldsymbol{I}_{N}
\end{array}\right)
$$

(where the asymptotic term $\mathcal{O}\left(1 / z^{m+1}\right)$ depends on $n$ ).

(Y4) As $z \rightarrow c, c \in\{a, b\}, c \neq \pm \infty$, we have

$$
\boldsymbol{Y}^{n}(z)=\left(\begin{array}{c:c}
\mathcal{O}(1) & \mathcal{O}(h(z)) \\
\hdashline \mathcal{O}(1) & \mathcal{O}(h(z))
\end{array}\right)
$$

where

$$
h(z)= \begin{cases}|z-c|^{\gamma_{c}}, & \text { if }-1<\gamma_{c}<0 \\ \log |z-c|, & \text { if } \gamma_{c}=0 \\ 1, & \text { if } \gamma_{c}>0\end{cases}
$$

Remark 2.1. Usually the asymptotic condition at infinity (2.3) is stated for $m=1$; however, it can be proved that $(2.3)$ for $m=1$ implies that this condition holds for every $m \in \mathbb{N}$.

Along with the $\mathrm{RH}$ problem $(\mathrm{Y} 1)-(\mathrm{Y} 4)$ we can consider the following dual problem: finding a matrix function $\boldsymbol{y}^{n}: \mathbb{C} \rightarrow \mathbb{C}^{2 N \times 2 N}$ such that

(y1) $\boldsymbol{y}^{n}$ is analytic in $\mathbb{C} \backslash[a, b]$.

(y2) $\boldsymbol{y}_{+}^{n}(x)=\left(\begin{array}{c:c}\boldsymbol{I}_{N} & -\boldsymbol{W}(x) \\ \hdashline \mathbf{0} & \boldsymbol{I}_{N}\end{array}\right) \boldsymbol{y}_{-}^{n}(x)$ when $x \in(a, b)$.

(y3) As $z \rightarrow \infty$, for every $m \in \mathbb{N}$ we have

$$
\boldsymbol{y}^{n}(z)=\left(\begin{array}{c:c}
z^{-n} \boldsymbol{I}_{N} & \mathbf{0} \\
\hdashline \mathbf{0} & z^{n} \boldsymbol{I}_{N}
\end{array}\right)\left(\boldsymbol{I}_{2 N}+\sum_{i=1}^{m} \frac{\tilde{\boldsymbol{Y}}_{i}^{n}}{z^{i}}+\mathcal{O}\left(1 / z^{m+1}\right)\right) .
$$

(y4) As $z \rightarrow c, c \in\{a, b\}, c \neq \pm \infty$, we have

$$
\boldsymbol{y}^{n}(z)=\left(\begin{array}{c:c}
\mathcal{O}(h(z)) & \mathcal{O}(h(z)) \\
\hdashline \mathcal{O}(1) & \mathcal{O}(1)
\end{array}\right)
$$

with $h$ defined in (2.4).

It will turn out that (y1)-(y4) is related to the inverse of the solution of (Y1)-(Y4).

For any integrable $N \times N$ matrix-valued function $\boldsymbol{F}$ on $[a, b]$,

$$
\mathcal{C}(\boldsymbol{F})(z):=\frac{1}{2 \pi i} \int_{a}^{b} \frac{\boldsymbol{F}(t)}{t-z} d t
$$

defines the Cauchy or Stieltjes transform of $\boldsymbol{F}$, which is a matrix-valued and analytic function in $\mathbb{C} \backslash[a, b]$. Let us introduce the matrix polynomials of the second kind (of degree $n-1$ ), defined by

$$
\boldsymbol{Q}_{n}(x)=\int_{a}^{b} \frac{\boldsymbol{P}_{n}(t)-\boldsymbol{P}_{n}(x)}{t-x} \boldsymbol{W}(t) d t, \quad n \geq 0 .
$$


We have

$$
2 \pi i \mathcal{C}\left(\boldsymbol{P}_{n} \boldsymbol{W}\right)(z)=\boldsymbol{Q}_{n}(z)+2 \pi i \boldsymbol{P}_{n}(z) \mathcal{C}(\boldsymbol{W})(z),
$$

where $2 \pi i \mathcal{C}(\boldsymbol{W})$ is the $m$-function of the weight matrix $\boldsymbol{W}$.

Finally, if $\boldsymbol{\kappa}_{n}$ is the leading coefficient of any corresponding normalized polynomial $\boldsymbol{P}_{n}$, we denote

$$
\gamma_{n}=\kappa_{n}^{*} \kappa_{n}
$$

Observe that a priori $\gamma_{n}$ depends on the selection of $\boldsymbol{\kappa}_{n}$.

The following is a complete analogue of the well-known theorem from [28], although its proof requires some additional considerations:

Theorem 2.2. The unique solution of the RHP (Y1)-(Y4) is

$$
\boldsymbol{Y}^{n}(z)=\boldsymbol{R}_{n}(z) \boldsymbol{Y}^{0}(z), \quad n \geq 0,
$$

where

$$
\boldsymbol{Y}^{0}(z)=\left(\begin{array}{c:c}
\boldsymbol{I}_{N} & \mathcal{C}(\boldsymbol{W})(z) \\
\hdashline \mathbf{0} & \boldsymbol{I}_{N}
\end{array}\right)
$$

and the transfer matrix $\boldsymbol{R}_{n}$ is a matrix polynomial given by $\boldsymbol{R}_{0}(z)=\boldsymbol{I}$,

$$
\boldsymbol{R}_{n}(z)=\left(\begin{array}{c:c}
\boldsymbol{\kappa}_{n}^{-1} \boldsymbol{P}_{n}(z) & \left(2 \pi i \boldsymbol{\kappa}_{n}\right)^{-1} \boldsymbol{Q}_{n}(z) \\
\hdashline-2 \pi i \boldsymbol{\kappa}_{n-1}^{*} \boldsymbol{P}_{n-1}(z) & -\boldsymbol{\kappa}_{n-1}^{*} \boldsymbol{Q}_{n-1}(z)
\end{array}\right), \quad n \in \mathbb{N} .
$$

Analogously, the unique solution of the RHP (y1)-(y4) is

$$
\boldsymbol{y}^{n}(z)=\boldsymbol{y}^{0}(z) \boldsymbol{r}_{n}(z), \quad n \geq 0,
$$

where

$$
\boldsymbol{y}^{0}(z)=\left(\begin{array}{c:c}
\boldsymbol{I}_{N} & -\mathcal{C}(\boldsymbol{W})(z) \\
\hdashline \mathbf{0} & \boldsymbol{I}_{N}
\end{array}\right)
$$

and the transfer matrix $\boldsymbol{r}_{n}$ is a matrix polynomial given by $\boldsymbol{r}_{0}(z)=\boldsymbol{I}$,

$$
\boldsymbol{r}_{n}(z)=\left(\begin{array}{c:c}
-\boldsymbol{Q}_{n-1}^{*}(z) \boldsymbol{\kappa}_{n-1} & -(2 \pi i)^{-1} \boldsymbol{Q}_{n}^{*}(z) \boldsymbol{\kappa}_{n}^{-*} \\
\hdashline 2 \pi i \boldsymbol{P}_{n-1}^{*}(z) \boldsymbol{\kappa}_{n-1} & \boldsymbol{P}_{n}^{*}(z) \boldsymbol{\kappa}_{n}^{-*}
\end{array}\right), \quad n \in \mathbb{N} .
$$

Moreover, for all $n \geq 0$,

$$
\begin{aligned}
& \boldsymbol{y}^{n}(z)=\left(\begin{array}{c:c}
\mathbf{0} & -\boldsymbol{I}_{N} \\
\hdashline \boldsymbol{I}_{N} & \mathbf{0}
\end{array}\right)\left(\boldsymbol{Y}^{n}(z)\right)^{T}\left(\begin{array}{c:c}
\mathbf{0} & \vdots \boldsymbol{I}_{N} \\
\hdashline-\boldsymbol{I}_{N} & \mathbf{0}
\end{array}\right)=\left(\boldsymbol{Y}^{n}(z)\right)^{-1}, \\
& \operatorname{det} \boldsymbol{Y}^{n}(z)=1, \quad \text { for all } z \in \mathbb{C} .
\end{aligned}
$$

Here $\boldsymbol{A}^{T}$ denotes the transpose of the matrix $\boldsymbol{A}$.

Remark 2.3. In the scalar case, when $\boldsymbol{Y}^{n}$ is a $2 \times 2$ matrix, there is no need to consider simultaneously both RHPs (Y1)-(Y4) and (y1)-(y4) since the existence of $\left(\boldsymbol{Y}^{n}\right)^{-1}$ and (2.11), (2.12) follow directly from $(2.13)$. In the general $(2 N) \times(2 N)$ case more care should be put in the analysis of the local behavior at the endpoints $a, b$, and (2.12) is no longer straightforward. 
Remark 2.4. The solution $\boldsymbol{Y}^{n}$ of (Y1)-(Y4) satisfies the following symmetry relation:

$$
\boldsymbol{Y}^{n}(z)=\left(\begin{array}{c:c}
\boldsymbol{I}_{N} & \mathbf{0} \\
\hdashline \mathbf{0} & -\boldsymbol{I}_{N}
\end{array}\right) \overline{\boldsymbol{Y}^{n}(\bar{z})}\left(\begin{array}{c:c}
\boldsymbol{I}_{N} & \mathbf{0} \\
\hdashline \mathbf{0} & -\dddot{\boldsymbol{I}}_{N}
\end{array}\right)
$$

which yields some obvious consequences for the block entries of $\boldsymbol{Y}^{n}$. This relation is established using the invariance of (Y1)-(Y4) by such a conjugation.

Remark 2.5. Alternatively, we can write the solution (2.8) as

$$
\boldsymbol{Y}^{n}(z)=\left(\begin{array}{c:c}
\widehat{\boldsymbol{P}}_{n}(z) & \mathcal{C}\left(\widehat{\boldsymbol{P}}_{n} \boldsymbol{W}\right)(z) \\
\hdashline-2 \pi i \boldsymbol{\gamma}_{n-1} \widehat{\boldsymbol{P}}_{n-1}(z) & -2 \pi i \boldsymbol{\gamma}_{n-1} \mathcal{C}\left(\widehat{\boldsymbol{P}}_{n-1} \boldsymbol{W}\right)(z)
\end{array}\right), \quad n \in \mathbb{N}
$$

where $\widehat{\boldsymbol{P}}_{n}$ denote the monic MOPRL of degree $n$ for the weight matrix $\boldsymbol{W}$. In the same vein,

$$
\left(\boldsymbol{Y}^{n}\right)^{-1}(z)=\left(\begin{array}{c:c}
-2 \pi i \mathcal{C}\left(\boldsymbol{W} \widehat{\boldsymbol{P}}_{n-1}^{*}\right)(z) \boldsymbol{\gamma}_{n-1} & -\mathcal{C}\left(\boldsymbol{W} \widehat{\boldsymbol{P}}_{n}^{*}\right)(z) \\
\hdashline 2 \pi i \widehat{\boldsymbol{P}}_{n-1}^{*}(z) \boldsymbol{\gamma}_{n-1} & \widehat{\boldsymbol{P}}_{n}^{*}(z)
\end{array}\right), \quad n \in \mathbb{N} .
$$

Remark 2.6. Although $\boldsymbol{\kappa}_{n}$ is defined up to a left unitary factor, the matrix coefficient $\gamma_{n}$ in (2.7) is unique. This is a consequence of the uniqueness of the solutions of the RHP above.

Remark 2.7. Formulas (2.8) and (2.10) show that generically the behavior of $\boldsymbol{Y}^{n}$ and $\left(\boldsymbol{Y}^{n}\right)^{-1}$ at the endpoints (and in general, any singular point) of the support of $\boldsymbol{W}$ is given by the local behavior of the Cauchy transform of the orthogonality weight, also known as its $m$-function, see $[50, \S 1.2]$.

Proof of Theorem 2.2. The fact that (2.8) or (2.15) is a solution of the RHP (Y1)-(Y4) is established following the proof for the scalar case, see e.g. [12], and taking into account that the orthogonality of $\widehat{\boldsymbol{P}}_{n}$ in (1.1) is equivalent to the homogeneous system

$$
\int_{a}^{b} x^{j} \widehat{\boldsymbol{P}}_{n}(x) \boldsymbol{W}(x) d x=\mathbf{0}, \quad j=0,1, \ldots, n-1 .
$$

The same applies to (2.10) or (2.16) and the RHP (y1)-(y4).

Consider the function $\boldsymbol{Z}^{n}(z)=\boldsymbol{Y}^{n}(z) \boldsymbol{y}^{n}(z)$; from (Y2) and (y2) it follows that it has no jump across $(a, b)$, and by (Y4), (y4),

$$
Z^{n}(z)=\mathcal{O}(h(z)), \quad z \rightarrow c \in\{a, b\} .
$$

Hence, $\boldsymbol{Z}^{n}$ has only removable singularities at the finite endpoints of the support of the weight, and thus is an entire function. It remains to observe that $\boldsymbol{Z}^{n}(\infty)=\boldsymbol{I}_{2 N}$ to conclude that $\boldsymbol{Z}^{n}(z)=\boldsymbol{I}_{2 N}$ for all $z \in \mathbb{C}$, which proves that $\boldsymbol{y}^{n}(z)=\left(\boldsymbol{Y}^{n}(z)\right)^{-1}$, as well as the uniqueness of both solutions. The first identity in (2.12) can be established by direct calculation or by observing that this transformation carries the RHP (Y1)-(Y4) to the RHP (y1)-(y4).

Finally, the scalar function $\operatorname{det} \boldsymbol{Y}^{n}(z)$ is analytic across $[a, b]$, and by $(2.8),(2.9)$,

$$
\operatorname{det} \boldsymbol{Y}^{n}(z)=\operatorname{det} \boldsymbol{R}_{n}(z)
$$

can have only removable singularities at the (finite) endpoints of the support of $\boldsymbol{W}$. Hence, $\operatorname{det} \boldsymbol{Y}^{n}(z)$ is an entire function; since by $(2.3), \operatorname{det} \boldsymbol{Y}^{n}(\infty)=1$, we conclude that it is identically 1 . 
Proposition 2.8. Let $\boldsymbol{P}_{n}=\boldsymbol{\kappa}_{n} \widehat{\boldsymbol{P}}_{n}, n \geq 0$, be a sequence of orthonormal MOPRL, and let $\left(\boldsymbol{Q}_{n}\right)_{n}$ be the corresponding matrix polynomials of the second kind (2.6). For these polynomials, define

$$
\boldsymbol{A}_{n}=\boldsymbol{\kappa}_{n-1} \boldsymbol{\kappa}_{n}^{-1} .
$$

Then

$$
\begin{aligned}
& \boldsymbol{Q}_{n}(z) \boldsymbol{P}_{n-1}^{*}(z)-\boldsymbol{P}_{n}(z) \boldsymbol{Q}_{n-1}^{*}(z)=\boldsymbol{A}_{n}^{-1}, \\
& \boldsymbol{Q}_{n}(z) \boldsymbol{P}_{n}^{*}(z)=\boldsymbol{P}_{n}(z) \boldsymbol{Q}_{n}^{*}(z) .
\end{aligned}
$$

Moreover, $\boldsymbol{P}_{n-1}^{*}(x) \boldsymbol{A}_{n} \boldsymbol{P}_{n}(x)$ and $\boldsymbol{Q}_{n-1}^{*}(x) \boldsymbol{A}_{n} \boldsymbol{Q}_{n}(x)$ are Hermitian for all $x \in \mathbb{R}$ and $n \geq 0$.

Remark 2.9. Identities (2.18) and (2.19) are also known as the Liouville-Ostrogradski formula and the Hermitian property, respectively. Both were originally derived for MOPRL in [20], using a different approach.

Proof. Using the explicit expressions for $\boldsymbol{Y}^{n}$ and $\left(\boldsymbol{Y}^{n}\right)^{-1}$ (written in the form (2.8) and (2.10)), from block entries $(1,1)$ and $(2,2)$ of the identity $\boldsymbol{Y}^{n}\left(\boldsymbol{Y}^{n}\right)^{-1}=\boldsymbol{I}$ we obtain the LiouvilleOstrogradski formula (2.18) while from block entries $(1,2)$ and $(2,1)$ we get the so-called Hermitian property (2.19).

Block entries $(1,2)$ and $(2,1)$ of $\left(\boldsymbol{Y}^{n}\right)^{-1} \boldsymbol{Y}^{n}=\boldsymbol{I}$ yield the commutativity relations

$$
\boldsymbol{P}_{n-1}^{*}(z) \boldsymbol{A}_{n} \boldsymbol{P}_{n}(z)=\boldsymbol{P}_{n}^{*}(z) \boldsymbol{A}_{n}^{*} \boldsymbol{P}_{n-1}(z), \quad \boldsymbol{Q}_{n-1}^{*}(z) \boldsymbol{A}_{n} \boldsymbol{Q}_{n}(z)=\boldsymbol{Q}_{n}^{*}(z) \boldsymbol{A}_{n}^{*} \boldsymbol{Q}_{n-1}(z),
$$

which show that $\boldsymbol{P}_{n-1}^{*}(x) \boldsymbol{A}_{n} \boldsymbol{P}_{n}(x)$ and $\boldsymbol{Q}_{n-1}^{*}(x) \boldsymbol{A}_{n} \boldsymbol{Q}_{n}(x)$ are Hermitian for all $x \in \mathbb{R}$ and $n \geq 0$.

For the formulation of the following results we need to introduce a new set of parameters,

$$
\boldsymbol{b}_{n, k}=\int_{a}^{b} x^{k} \widehat{\boldsymbol{P}}_{n}(x) \boldsymbol{W}(x) d x, \quad k=n, n+1, \ldots,
$$

where $\widehat{\boldsymbol{P}}_{n}$ are the monic MOPRL. In the spirit of [48], we can express them in terms of the coefficients $\boldsymbol{a}_{n, j}$ of the polynomials $\widehat{\boldsymbol{P}}_{n}$ (see (1.1)) in a form suitable for numerical implementation:

Lemma 2.10. Let $\boldsymbol{\Omega}$ be the block lower triangular matrix built up from the coefficients of the $\operatorname{MOPRL}\left(\widehat{\boldsymbol{P}}_{n}\right)_{n}$,

$$
\boldsymbol{\Omega}=\left(\begin{array}{cccc}
\boldsymbol{I} & & & \\
\boldsymbol{a}_{1,0} & \boldsymbol{I} & & \\
\vdots & \vdots & \ddots & \\
\boldsymbol{a}_{n, 0} & \boldsymbol{a}_{n, 1} & \cdots & \boldsymbol{I}
\end{array}\right), \quad \text { so that }\left(\begin{array}{c}
\widehat{\boldsymbol{P}}_{0}(x) \\
\widehat{\boldsymbol{P}}_{1}(x) \\
\vdots \\
\widehat{\boldsymbol{P}}_{n}(x)
\end{array}\right)=\boldsymbol{\Omega}\left(\begin{array}{c}
\boldsymbol{I} \\
x \boldsymbol{I} \\
\vdots \\
x^{n} \boldsymbol{I}
\end{array}\right), \quad \boldsymbol{I}=\boldsymbol{I}_{N}
$$

Then $\boldsymbol{b}_{n, k}$ defined in (2.20) can be obtained from the last $N \times N$ block row of $\boldsymbol{\Omega}^{-1}$ as follows:

$$
\boldsymbol{b}_{n-k, n}^{*} \gamma_{n-k}=\left(\boldsymbol{\Omega}^{-1}\right)_{n+1, n-k+1}, \quad k=0, \ldots, n .
$$

Proof. Observe that

$$
\begin{aligned}
\left(\boldsymbol{b}_{0, n}^{*}, \ldots, \boldsymbol{b}_{n-1, n}^{*}, \boldsymbol{b}_{n, n}^{*}\right) & =\int_{a}^{b} x^{n} \boldsymbol{W}\left(\widehat{\boldsymbol{P}}_{0}^{*}, \widehat{\boldsymbol{P}}_{1}^{*}, \ldots, \widehat{\boldsymbol{P}}_{n}^{*}\right) d x \\
& =\int_{a}^{b} x^{n} \boldsymbol{W}\left(\boldsymbol{I}, x \boldsymbol{I}, \ldots, x^{n} \boldsymbol{I}\right) \boldsymbol{\Omega}^{*} d x=\left(\boldsymbol{\mu}_{n}, \boldsymbol{\mu}_{n+1}, \ldots, \boldsymbol{\mu}_{2 n}\right) \boldsymbol{\Omega}^{*}
\end{aligned}
$$


where $\boldsymbol{\mu}_{n}=\int_{a}^{b} x^{n} \boldsymbol{W}(x) d x$ are the moments of the weight matrix $\boldsymbol{W}$. Analogously, by (1.2),

$$
\begin{aligned}
& \left(\operatorname{diag}\left(\gamma_{k}\right)_{k=0}^{n}\right)^{-1}=\operatorname{diag}\left(\gamma_{k}^{-1}\right)_{k=0}^{n}=\int_{a}^{b}\left(\begin{array}{c}
\widehat{\boldsymbol{P}}_{0} \\
\widehat{\boldsymbol{P}}_{1} \\
\vdots \\
\widehat{\boldsymbol{P}}_{n}
\end{array}\right) \boldsymbol{W}\left(\widehat{\boldsymbol{P}}_{0}^{*}, \widehat{\boldsymbol{P}}_{1}^{*}, \ldots, \widehat{\boldsymbol{P}}_{n}^{*}\right) d x \\
& \quad=\boldsymbol{\Omega} \int_{a}^{b}\left(\begin{array}{c}
\boldsymbol{I} \\
x \boldsymbol{I} \\
\vdots \\
x^{n} \boldsymbol{I}
\end{array}\right) \boldsymbol{W}\left(\boldsymbol{I}, x \boldsymbol{I}, \ldots, x^{n} \boldsymbol{I}\right) \boldsymbol{\Omega}^{*}=\boldsymbol{\Omega}\left(\begin{array}{cccc}
\boldsymbol{\mu}_{0} & \boldsymbol{\mu}_{1} & \cdots & \boldsymbol{\mu}_{n} \\
\boldsymbol{\mu}_{1} & \boldsymbol{\mu}_{2} & \cdots & \boldsymbol{\mu}_{n+1} \\
\vdots & \vdots & \ddots & \vdots \\
\boldsymbol{\mu}_{n} & \boldsymbol{\mu}_{n+1} & \cdots & \boldsymbol{\mu}_{2 n}
\end{array}\right) \boldsymbol{\Omega}^{*}
\end{aligned}
$$

Hence,

$$
\begin{aligned}
& \left(\boldsymbol{b}_{0, n}^{*} \gamma_{0}, \ldots, \boldsymbol{b}_{n-1, n}^{*} \gamma_{n-1}, \boldsymbol{b}_{n, n}^{*} \gamma_{n}\right) \\
& \quad=\left(\boldsymbol{\mu}_{n}, \boldsymbol{\mu}_{n+1}, \ldots, \boldsymbol{\mu}_{2 n}\right)\left(\begin{array}{cccc}
\boldsymbol{\mu}_{0} & \boldsymbol{\mu}_{1} & \cdots & \boldsymbol{\mu}_{n} \\
\boldsymbol{\mu}_{1} & \boldsymbol{\mu}_{2} & \cdots & \boldsymbol{\mu}_{n+1} \\
\vdots & \vdots & \ddots & \vdots \\
\boldsymbol{\mu}_{n} & \boldsymbol{\mu}_{n+1} & \cdots & \boldsymbol{\mu}_{2 n}
\end{array}\right) \quad \boldsymbol{\Omega}^{-1}=(\mathbf{0}, \mathbf{0}, \ldots, \boldsymbol{I}) \boldsymbol{\Omega}^{-1},
\end{aligned}
$$

which yields (2.21).

For what follows it is useful to single out the explicit expressions for $\boldsymbol{b}_{n-3, n}, \ldots, \boldsymbol{b}_{n, n}$, that are obtained from Lemma 2.10 by direct computations:

Corollary 2.11. Coefficients $\boldsymbol{b}_{n-k, n}$, for $k=0,1,2,3$, are given by

$$
\begin{aligned}
& \gamma_{n} \boldsymbol{b}_{n, n}=\boldsymbol{I}, \\
& \gamma_{n-1} \boldsymbol{b}_{n-1, n}=-\boldsymbol{a}_{n, n-1}^{*}, \\
& \gamma_{n-2} \boldsymbol{b}_{n-2, n}=\boldsymbol{a}_{n-1, n-2}^{*} \boldsymbol{a}_{n, n-1}^{*}-\boldsymbol{a}_{n, n-2}^{*}, \\
& \gamma_{n-3} \boldsymbol{b}_{n-3, n}=-\boldsymbol{a}_{n-2, n-3}^{*} \boldsymbol{a}_{n-1, n-2}^{*} \boldsymbol{a}_{n, n-1}^{*}+\boldsymbol{a}_{n-1, n-3}^{*} \boldsymbol{a}_{n, n-1}^{*}+\boldsymbol{a}_{n-2, n-3}^{*} \boldsymbol{a}_{n, n-2}^{*}-\boldsymbol{a}_{n, n-3}^{*} .
\end{aligned}
$$

Now we return to Theorem 2.2; as its immediate consequence we can relate the coefficients in the asymptotic expansion of $\boldsymbol{Y}^{n}$ and of $\left(\boldsymbol{Y}^{n}\right)^{-1}$, with the coefficients $\boldsymbol{a}_{n, j}$ and $\boldsymbol{b}_{n, j}$ :

Corollary 2.12. The coefficients $\boldsymbol{Y}_{i}^{n}$ in (2.3) are given by

$$
\boldsymbol{Y}_{i}^{n}=\left(\begin{array}{c:c}
\boldsymbol{a}_{n, n-i} & -\frac{1}{2 \pi i} \boldsymbol{b}_{n, n+i-1} \\
\hdashline-2 \pi i \gamma_{n-1} \boldsymbol{a}_{n-1, n-i} & \gamma_{n-1} \boldsymbol{b}_{n-1, n+i-1}
\end{array}\right), \quad i \geq 0
$$

Analogously, the coefficients $\tilde{\boldsymbol{Y}}_{i}^{n}$ in (2.5) are given by

$$
\tilde{\boldsymbol{Y}}_{i}^{n}=\left(\begin{array}{c:c}
\boldsymbol{b}_{n-1, n+i-1}^{T} \boldsymbol{\gamma}_{n-1} & \frac{1}{2 \pi i} \boldsymbol{b}_{n, n+i-1}^{T} \\
\hdashline 2 \pi i \boldsymbol{a}_{n-1, n-i}^{T} \boldsymbol{\gamma}_{n-1} & \boldsymbol{a}_{n, n-i}^{T}
\end{array}\right), \quad i \geq 0 .
$$

Remark 2.13. One of the consequences of (2.14) is that $\boldsymbol{a}_{n, j}$ and $\boldsymbol{b}_{n, j}$ appearing in (2.22), (2.23) belong to $\mathbb{R}^{N \times N}$.

The explicit expressions for coefficients of the asymptotic expansion above, combined with the obvious fact that $\boldsymbol{Y}^{n}\left(\boldsymbol{Y}^{n}\right)^{-1}=\boldsymbol{I}_{2 N}$, yield in a straightforward way the following identities: 
Proposition 2.14. The coefficients of the monic MOPRL $\widehat{\boldsymbol{P}}_{n}$ and the coefficients of the Cauchy transform $\mathcal{C}\left(\widehat{\boldsymbol{P}}_{n} \boldsymbol{W}\right)$ (i.e. the sequences $\left.\boldsymbol{b}_{n, k}\right)$ satisfy the following relations

$$
\sum_{j=0}^{m} \boldsymbol{a}_{n, n-m+j} \boldsymbol{b}_{n-1, n+j-1}^{*}=\sum_{j=0}^{m} \boldsymbol{b}_{n, n+m-j-1} \boldsymbol{a}_{n-1, n-j}^{*}, \quad m \geq 1,
$$

and

$$
\sum_{j=0}^{m} \boldsymbol{a}_{n, n-m+j} \boldsymbol{b}_{n, n+j-1}^{*}=\sum_{j=0}^{m} \boldsymbol{b}_{n, n+m-j-1} \boldsymbol{a}_{n, n-j}^{*}, \quad m \geq 1 .
$$

In particular, for $m=1$ we recover the second identity of Corollary 2.11 .

Proof. From the asymptotic behavior of $\boldsymbol{Y}^{n}$ and $\left(\boldsymbol{Y}^{n}\right)^{-1}$ and using $\boldsymbol{Y}^{n}\left(\boldsymbol{Y}^{n}\right)^{-1}=\boldsymbol{I}$ we get

$$
\sum_{j=0}^{m} \boldsymbol{Y}_{m-j}^{n} \tilde{\boldsymbol{Y}}_{j}^{n}=\mathbf{0}, \quad m \geq 1
$$

from which block entries $(1,1)$ and $(1,2)$ give $(2.24)$ and $(2.25)$, respectively.

We finish this section with the matrix analogue of the Christoffel-Darboux (CD) formula for MOPRL, written in terms of the solution of the characterizing RHP. For an orthonormal family $\left(\boldsymbol{P}_{n}\right)_{n}$ we consider the kernel

$$
\boldsymbol{K}_{n}(x, y)=\sum_{j=0}^{n-1} \boldsymbol{P}_{j}^{*}(y) \boldsymbol{P}_{j}(x), \quad x, y \in \mathbb{R} .
$$

Then the CD formula (see [20]) reads as

$$
\boldsymbol{K}_{n}(x, y)=\frac{\boldsymbol{P}_{n-1}^{*}(y) \boldsymbol{A}_{n} \boldsymbol{P}_{n}(x)-\boldsymbol{P}_{n}^{*}(y) \boldsymbol{A}_{n}^{*} \boldsymbol{P}_{n-1}(x)}{x-y} .
$$

Proposition 2.15. The matrix CD kernel (2.26) satisfies, for $x, y \in \mathbb{R}$,

$$
\begin{aligned}
& \boldsymbol{K}_{n}(x, y)=-\frac{1}{2 \pi i(x-y)}\left(\boldsymbol{Y}_{21}^{*}(y) \boldsymbol{Y}_{11}(x)-\boldsymbol{Y}_{11}^{*}(y) \boldsymbol{Y}_{21}(x)\right) \\
& =\frac{1}{2 \pi i(x-y)}\left(\begin{array}{ll}
\mathbf{0} & \boldsymbol{I}
\end{array}\right)\left(\boldsymbol{Y}^{n}\right)_{+}^{-1}(y)\left(\boldsymbol{Y}^{n}\right)_{+}(x)\left(\begin{array}{l}
\boldsymbol{I} \\
\mathbf{0}
\end{array}\right) .
\end{aligned}
$$

The proof follows taking into account the Christoffel-Darboux formula (2.27), the expression for $\boldsymbol{A}_{n}$ in (2.17) and the expression of the inverse $\left(\boldsymbol{Y}^{n}\right)^{-1}$ in (2.16). The last proposition for general weight matrices of rectangular size can be found in [18].

\subsection{Difference and differential relations}

Both the MOPRL and the solution of the RHP given in Theorem 2.2 depend on two variables, the discrete $n$ and the continuous $z$. We can derive further properties by analyzing the variation of this solution with respect to either variable, which yields linear relations of the form

$$
\boldsymbol{Y}^{n+1}(z)=\boldsymbol{E}_{n}(z) \boldsymbol{Y}^{n}(z), \quad \frac{d}{d z} \boldsymbol{Y}^{n}(z)=\boldsymbol{F}_{n}(z) \boldsymbol{Y}^{n}(z) .
$$

The general methodology to get these equations may be traced back to the original work of Gelfand, Levitan and other authors. The idea is that if the jump matrix for a RHP is independent of a variable, then a variation with respect to that variable leads to an identity. The fact that the jump matrix in (2.2) is independent of $n$ allows one to obtain immediately the first identity in (2.28), which in turn is connected to the well-known three-term recurrence relation for MOPRL. 
Theorem 2.16. There is a unique sequence of matrix coefficients $\boldsymbol{\alpha}_{n}$ such that the solution of the $\operatorname{RHP}^{n}(z)$ satisfies the following first-order difference equation

$$
\boldsymbol{Y}^{n+1}(z)=\left(\begin{array}{c:c}
z \boldsymbol{I}-\boldsymbol{\alpha}_{n} & \frac{1}{2 \pi i} \boldsymbol{\gamma}_{n}^{-1} \\
\hdashline-2 \pi i \gamma_{n} & \mathbf{0}
\end{array}\right) \boldsymbol{Y}^{n}(z)
$$

Consequently, the monic MOPRL $\widehat{\boldsymbol{P}}_{n}$ satisfy the following three-term recurrence relation:

$$
x \widehat{\boldsymbol{P}}_{n}(x)=\widehat{\boldsymbol{P}}_{n+1}(x)+\boldsymbol{\alpha}_{n} \widehat{\boldsymbol{P}}_{n}(x)+\boldsymbol{\beta}_{n} \widehat{\boldsymbol{P}}_{n-1}(x), \quad n \geq 0 .
$$

The coefficients $\boldsymbol{\alpha}_{n}$ and $\boldsymbol{\beta}_{n}$, as well as $\boldsymbol{\gamma}_{n}$ (defined in (2.7)) can be expressed either in terms of the elements of the solution of the RHP or in terms of the coefficients $\boldsymbol{a}_{n, j}$ (see (1.1)) as follows:

$$
\begin{aligned}
& \boldsymbol{\alpha}_{n}=\left(\boldsymbol{Y}_{1}^{n}\right)_{11}-\left(\boldsymbol{Y}_{1}^{n+1}\right)_{11}=\boldsymbol{a}_{n, n-1}-\boldsymbol{a}_{n+1, n}, \quad \boldsymbol{\beta}_{n}=\left(\boldsymbol{Y}_{1}^{n}\right)_{12}\left(\boldsymbol{Y}_{1}^{n}\right)_{21}=\boldsymbol{\gamma}_{n}^{-1} \boldsymbol{\gamma}_{n-1}, \\
& \boldsymbol{\gamma}_{n}=-\frac{1}{2 \pi i}\left(\boldsymbol{Y}_{1}^{n+1}\right)_{21}=-\frac{1}{2 \pi i}\left(\boldsymbol{Y}_{1}^{n}\right)_{12}^{-1} .
\end{aligned}
$$

Proof. The matrix-valued function $\boldsymbol{R}=\boldsymbol{Y}^{n+1}\left(\boldsymbol{Y}^{n}\right)^{-1}$, analytic in $\mathbb{C} \backslash[a, b]$, satisfies $\boldsymbol{R}_{+}(x)=$ $\boldsymbol{R}_{-}(x)$ for all $x \in(a, b)$. By the Morera's theorem, $\boldsymbol{R}$ is analytic in $\mathbb{C} \backslash\{a, b\}$. The behavior at the exceptional points gives that the singularities at $a$ and $b$ are removable, and $\boldsymbol{R}$ is an entire function. From (2.3) and Liouville's theorem we conclude that

$$
\boldsymbol{Y}^{n+1}(z)=\left(\begin{array}{c:c}
z \boldsymbol{I}+\left(\boldsymbol{Y}_{1}^{n+1}\right)_{11}-\left(\boldsymbol{Y}_{1}^{n}\right)_{11} & -\left(\boldsymbol{Y}_{1}^{n}\right)_{12} \\
\hdashline\left(\boldsymbol{Y}_{1}^{n+1}\right)_{21} & \mathbf{0}
\end{array}\right) \boldsymbol{Y}^{n}(z) .
$$

This proves (2.29) along with the expression for $\boldsymbol{\alpha}_{n}$ in terms of the solution of the RHP. The three term recurrence relation (2.30) for the MOPRL is obtained by considering the $(1,1)$ block entry of (2.32). Finally, the rest of the expressions for the coefficients is found using Corollary 2.12 (formula $(2.22)$ ).

We turn now to the dependence of $\boldsymbol{Y}^{n}$ on the continuous variable $z$; notice however that the jump (2.2) along the real line does depend on this variable, so the application of the paradigm explained at the beginning of this subsection is not straightforward. At this stage we can only aspire to find a differential relation like in (2.28), but with a matrix $\boldsymbol{F}_{n}$ depending upon the entries of $\boldsymbol{Y}^{n}$ (or equivalently, upon the MOPRL themselves).

Theorem 2.17. The solution of the RHP for $\boldsymbol{Y}^{n}(z)$ satisfies the following first-order matrix differential equation

$$
\frac{d}{d z} \boldsymbol{Y}^{n}(z)=\left(\begin{array}{c:c}
-\mathfrak{B}_{n}(z) & -\frac{1}{2 \pi i} \gamma_{n}^{-1} \mathfrak{A}_{n}(z) \\
\hdashline 2 \pi i \mathfrak{A}_{n-1}(z) \gamma_{n-1} & \mathfrak{B}_{n}^{*}(z)
\end{array}\right) \boldsymbol{Y}^{n}(z),
$$

where

$$
\mathfrak{A}_{n}(z)=-\gamma_{n}\left(\int_{a}^{b} \frac{\widehat{\boldsymbol{P}}_{n}(t) \boldsymbol{W}^{\prime}(t) \widehat{\boldsymbol{P}}_{n}^{*}(t)}{t-z} d t-\left.\frac{\widehat{\boldsymbol{P}}_{n}(t) \boldsymbol{W}(t) \widehat{\boldsymbol{P}}_{n}^{*}(t)}{t-z}\right|_{t=a} ^{t=b}\right)
$$

and

$$
\mathfrak{B}_{n}(z)=-\left(\int_{a}^{b} \frac{\widehat{\boldsymbol{P}}_{n}(t) \boldsymbol{W}^{\prime}(t) \widehat{\boldsymbol{P}}_{n-1}^{*}(t)}{t-z} d t-\left.\frac{\widehat{\boldsymbol{P}}_{n}(t) \boldsymbol{W}(t) \widehat{\boldsymbol{P}}_{n-1}^{*}(t)}{t-z}\right|_{t=a} ^{t=b}\right) \boldsymbol{\gamma}_{n-1},
$$

provided that all the functions in the right-hand sides of (2.34) and (2.35) exist for $z \in \mathbb{C} \backslash[a, b]$. In particular,

$$
\gamma_{n} \mathfrak{A}_{n}^{*}(z)=\mathfrak{A}_{n}(z) \gamma_{n} .
$$


Remark 2.18. Formulas (2.34) and (2.35) were introduced in the scalar case in [7] and in the matrix case (for a normalized family) in [25]. In general, they bear a formal character. In particular, the boundary terms in (2.34) and (2.35) may be undefined, depending on the behavior of $\boldsymbol{W}$ at the endpoints (see (2.1)). As it will be shown in the next section, these formulas simplify considerably after considering a special transformation of the RHP for $\boldsymbol{Y}^{n}$. Finally observe that the coefficients (2.34) and (2.35) are unique since $\boldsymbol{Y}^{n}$ is unique.

Proof. Consider the matrix-valued function $\boldsymbol{R}^{n}(z)=\left[\frac{d}{d z} \boldsymbol{Y}^{n}(z)\right]\left(\boldsymbol{Y}^{n}\right)^{-1}(z)$. From the explicit expressions (2.15) and (2.16) we have that the block entries of $\boldsymbol{R}^{n}$ are

$$
\begin{aligned}
& \left(\boldsymbol{R}^{n}(z)\right)_{11}=-2 \pi i\left(\widehat{\boldsymbol{P}}_{n}^{\prime}(z) \mathcal{C}\left(\boldsymbol{W} \widehat{\boldsymbol{P}}_{n-1}^{*}\right)(z)-\mathcal{C}\left(\widehat{\boldsymbol{P}}_{n} \boldsymbol{W}\right)^{\prime}(z) \widehat{\boldsymbol{P}}_{n-1}^{*}(z)\right) \boldsymbol{\gamma}_{n-1}, \\
& \left(\boldsymbol{R}^{n}(z)\right)_{12}=-\widehat{\boldsymbol{P}}_{n}^{\prime}(z) \mathcal{C}\left(\boldsymbol{W} \widehat{\boldsymbol{P}}_{n}^{*}\right)(z)+\mathcal{C}\left(\widehat{\boldsymbol{P}}_{n} \boldsymbol{W}\right)^{\prime}(z) \widehat{\boldsymbol{P}}_{n}^{*}(z), \\
& \left(\boldsymbol{R}^{n}(z)\right)_{21}=(2 \pi i)^{2} \boldsymbol{\gamma}_{n-1}\left(\widehat{\boldsymbol{P}}_{n-1}^{\prime}(z) \mathcal{C}\left(\boldsymbol{W} \widehat{\boldsymbol{P}}_{n-1}^{*}\right)(z)-\mathcal{C}\left(\widehat{\boldsymbol{P}}_{n-1} \boldsymbol{W}\right)^{\prime}(z) \widehat{\boldsymbol{P}}_{n-1}^{*}(z)\right) \boldsymbol{\gamma}_{n-1}, \\
& \left(\boldsymbol{R}^{n}(z)\right)_{22}=-2 \pi i \boldsymbol{\gamma}_{n-1}\left(-\widehat{\boldsymbol{P}}_{n-1}^{\prime}(z) \mathcal{C}\left(\boldsymbol{W} \widehat{\boldsymbol{P}}_{n}^{*}\right)(z)+\mathcal{C}\left(\widehat{\boldsymbol{P}}_{n-1} \boldsymbol{W}\right)^{\prime}(z) \widehat{\boldsymbol{P}}_{n}^{*}(z)\right) .
\end{aligned}
$$

In particular, $-\left(\boldsymbol{R}^{n}(z)\right)_{11}=\left(\boldsymbol{R}^{n}(z)\right)_{22}^{*}$ and $-2 \pi i \boldsymbol{\gamma}_{n}\left(\boldsymbol{R}^{n}(z)\right)_{12}=\frac{1}{2 \pi i}\left(\boldsymbol{R}^{n+1}(z)\right)_{21} \boldsymbol{\gamma}_{n}^{-1}$.

We will now derive formulas (2.34) and (2.35). For that purpose we need the following technical observations:

\section{Lemma 2.19.}

(i) For every $\boldsymbol{P} \in \mathbb{P}_{n}$,

$$
\boldsymbol{P}(z) \mathcal{C}\left(\boldsymbol{W} \widehat{\boldsymbol{P}}_{n}^{*}\right)(z)=\mathcal{C}\left(\boldsymbol{P} \boldsymbol{W} \widehat{\boldsymbol{P}}_{n}^{*}\right)(z), \quad \mathcal{C}\left(\widehat{\boldsymbol{P}}_{n} \boldsymbol{W}\right)(z) \boldsymbol{P}(z)=\mathcal{C}\left(\widehat{\boldsymbol{P}}_{n} \boldsymbol{W} \boldsymbol{P}\right)(z) .
$$

(ii) For any differentiable and integrable matrix function $\boldsymbol{F}$ bounded at $z=a, b$,

$$
\frac{d}{d z} \mathcal{C}(\boldsymbol{F})(z)=\mathcal{C}\left(\boldsymbol{F}^{\prime}\right)-\left.\frac{1}{2 \pi i} \frac{\boldsymbol{F}(t)}{t-z}\right|_{t=a} ^{t=b}, \quad z \in \mathbb{C} \backslash[a, b]
$$

Identities in $(i)$ follow by adding and subtracting $\mathcal{C}\left(\boldsymbol{P} \boldsymbol{W} \widehat{\boldsymbol{P}}_{n}^{*}\right)(z)$ and using the orthogonality of the MOPRL, and $(i i)$ is obtained by integration by parts and using that $\frac{d}{d z}(1 /(t-z))=$ $-\frac{d}{d t}(1 /(t-z))$.

We return to the proof of Theorem 2.17, showing in detail how to obtain $\mathfrak{B}_{n}(z)$. For $\mathfrak{A}_{n}(z)$ the computations are similar and will be omitted for the sake of brevity.

Using $(i)$ from the previous lemma and the product rule of differentiation we get

$$
\left(\boldsymbol{R}^{n}(z)\right)_{11}=-2 \pi i\left(\mathcal{C}\left(\widehat{\boldsymbol{P}}_{n}^{\prime} \boldsymbol{W} \widehat{\boldsymbol{P}}_{n-1}^{*}\right)(z)-\mathcal{C}\left(\widehat{\boldsymbol{P}}_{n} \boldsymbol{W} \widehat{\boldsymbol{P}}_{n-1}^{*}\right)^{\prime}(z)+\mathcal{C}\left(\widehat{\boldsymbol{P}}_{n} \boldsymbol{W}\left(\widehat{\boldsymbol{P}}_{n-1}^{*}\right)^{\prime}\right)(z)\right) \boldsymbol{\gamma}_{n-1} .
$$

Applying now $(i i)$ to the second term and canceling we get

$$
\left(\boldsymbol{R}^{n}(z)\right)_{11}=-2 \pi i\left(-\mathcal{C}\left(\widehat{\boldsymbol{P}}_{n} \boldsymbol{W}^{\prime} \widehat{\boldsymbol{P}}_{n-1}^{*}\right)(z)+\left.\frac{1}{2 \pi i} \frac{\widehat{\boldsymbol{P}}_{n}(t) \boldsymbol{W}(t) \widehat{\boldsymbol{P}}_{n-1}^{*}(t)}{t-z}\right|_{t=a} ^{t=b}\right) \boldsymbol{\gamma}_{n-1},
$$

which yields (2.35).

Let us discuss now two main consequences of the existence of the first order matrix-valued differential equation (2.33).

One one hand, equations (2.28), known as the Lax pair for the RHP, are clearly overdetermined, so compatibility conditions (via cross-differentiation of both equations) yield

$$
\boldsymbol{E}_{n}^{\prime}(z)+\boldsymbol{E}_{n}(z) \boldsymbol{F}_{n}(z)=\boldsymbol{F}_{n+1}(z) \boldsymbol{E}_{n}(z)
$$


also known as string equations. They allow us, for instance, to recover the sequence $\left\{\boldsymbol{F}_{n}\right\}$ from the sequence $\left\{\boldsymbol{E}_{n}\right\}$ and the initial value $\boldsymbol{F}_{1}$. These ideas relate also the RHP to nonlinear problems such as the nonlinear Schrödinger equation (NLS), see [17], and the Toda flow, see [27]. In our situation, the compatibility conditions (2.37) considered entry-wise imply the following:

Proposition 2.20. The recurrence relations

$$
\boldsymbol{I}+\mathfrak{B}_{n+1}(z)\left(z \boldsymbol{I}-\boldsymbol{\alpha}_{n}\right)-\left(z \boldsymbol{I}-\boldsymbol{\alpha}_{n}\right) \mathfrak{B}_{n}(z)=\mathfrak{A}_{n+1}^{*}(z) \boldsymbol{\beta}_{n+1}-\boldsymbol{\beta}_{n} \mathfrak{A}_{n-1}^{*}(z)
$$

and

$$
\mathfrak{B}_{n+1}(z)+\boldsymbol{\gamma}_{n}^{-1} \mathfrak{B}_{n}^{*}(z) \boldsymbol{\gamma}_{n}=\left(z \boldsymbol{I}-\boldsymbol{\alpha}_{n}\right) \mathfrak{A}_{n}^{*}(z)
$$

hold for every $n \geq 0$, where $\boldsymbol{\alpha}_{n}$ and $\boldsymbol{\beta}_{n}$ are the coefficients of the three-term recurrence relation (2.30).

Proof. Block entries $(1,1)$ and $(1,2)$ of $(2.37)$ give (2.38) and (2.39), respectively. Block entry $(2,1)$ of (2.37) is equivalent to (2.39) using relation (2.36) and $\boldsymbol{\alpha}_{n}=\boldsymbol{\gamma}_{n}^{-1} \boldsymbol{\alpha}_{n}^{*} \boldsymbol{\gamma}_{n}$ (which is a consequence of substituting (2.22) for $i=2$ in (2.29) as $z \rightarrow \infty$, along with (2.31)), while block entry $(2,2)$ is equivalent to $(2.38)$ as well.

Another consequence of Theorem 2.17 is the existence of the ladder operators for MOPRL:

Corollary 2.21. The monic MOPRL $\left(\widehat{\boldsymbol{P}}_{n}\right)_{n}$ satisfy the following difference-differential relations (lowering and raising operators, respectively):

$$
\widehat{\boldsymbol{P}}_{n}^{\prime}(z)=-\mathfrak{B}_{n}(z) \widehat{\boldsymbol{P}}_{n}(z)+\mathfrak{A}_{n}^{*}(z) \boldsymbol{\beta}_{n} \widehat{\boldsymbol{P}}_{n-1}(z)
$$

and

$$
\widehat{\boldsymbol{P}}_{n}^{\prime}(z)=\left[\mathfrak{A}_{n}^{*}(z)\left(z \boldsymbol{I}-\boldsymbol{\alpha}_{n}\right)-\mathfrak{B}_{n}(z)\right] \widehat{\boldsymbol{P}}_{n}(z)-\mathfrak{A}_{n}^{*}(z) \widehat{\boldsymbol{P}}_{n+1}(z) .
$$

Proof. Block entry $(1,1)$ of $(2.33)$ gives the lowering operator $(2.40)$, using the expression for $\boldsymbol{\beta}_{n}$ in Theorem 2.16 , while block entry $(2,1)$ of $(2.33)$ gives the raising operator $(2.41)$ using $(2.36)$ and (2.39).

Proposition 2.20 and Corollary 2.21 were already known for the normalized MOPRL in [25]; the RH approach gives an alternative proof of these results. The ladder operators are the basic differential relations for MOPRL. It is well-known that they can be combined to build a secondorder differential equation satisfied by the polynomials. This fact was mentioned in [25], but no explicit expression of the differential equation was given; here we present it for completeness:

Corollary 2.22. The polynomials $\left(\widehat{\boldsymbol{P}}_{n}\right)_{n}$ satisfy the following second-order differential equation

$$
\widehat{\boldsymbol{P}}_{n}^{\prime \prime}(z)+\mathfrak{M}_{n}(z) \widehat{\boldsymbol{P}}_{n}^{\prime}(z)+\mathfrak{N}_{n}(z) \widehat{\boldsymbol{P}}_{n}(z)=\mathbf{0},
$$

where

$$
\mathfrak{M}_{n}(z)=-\left(\mathfrak{A}_{n}^{*}(z)\right)^{\prime} \mathfrak{A}_{n}^{-*}(z)+\mathfrak{B}_{n}(z)-\mathfrak{A}_{n}^{*}(z)\left(z \boldsymbol{I}-\boldsymbol{\alpha}_{n}\right)+\mathfrak{A}_{n}^{*}(z) \mathfrak{B}_{n+1}(z) \mathfrak{A}_{n}^{-*}(z)
$$

and

$$
\mathfrak{N}_{n}(z)=\mathfrak{M}_{n}(z) \mathfrak{B}_{n}(z)-\mathfrak{B}_{n}^{2}(z)+\mathfrak{B}_{n}^{\prime}(z)+\mathfrak{A}_{n}^{*}(z) \boldsymbol{\beta}_{n} \mathfrak{A}_{n-1}^{*}(z),
$$

provided that the inverse of $\mathfrak{A}_{n}^{*}(z)$ exists for $z \in \mathbb{C} \backslash[a, b]$. Here and below, the superscript -* denotes the conjugate transpose of the inverse. 
Proof. Differentiate the lowering operator (2.40) and substitute the raising operator (2.41) evaluated at $n-1$.

Remark 2.23. Note that we can easily obtain another second-order differential operator satisfied by the orthogonal polynomials reversing the ladder operators. This new differential equation needs not in principle be the same as (2.42). Nevertheless, it is straightforward to see that both equations are equivalent using the compatibility conditions (2.38) and (2.39).

Although Theorem 2.17 gives explicit expressions of $\mathfrak{A}_{n}(z)$ and $\mathfrak{B}_{n}(z)$, these coefficients are usually difficult to calculate. They are not even defined on the interval $[a, b]$. In the next section we will introduce some additional assumptions that simplify the differential equation considerably.

\section{Transformation of the RHP when $\operatorname{supp}(W)=\mathbb{R}$}

As it was pointed out in the previous section, the independence of the jump in (2.2) with respect to the discrete variable $n$ allows one to obtain the three-term recurrence relation in a straightforward way. Under additional assumptions on the jumps we can perform a transformation of the RHP in such a way that a new jump is independent of the continuous variable $z$. This will have consequences on the resulting differential relations.

In the matrix case there is some extra freedom absent in the scalar situation, that gives us a whole new family of differential relations, and consequently, a whole class of ladder operators, some of them of the 0 -th order, something that is not possible in the scalar case. The combination of the ladder operators will give rise to a class of second-order differential equations, some of them of order less than 2.

For simplicity, we will focus here on the case when $[a, b]=\mathbb{R}$, assuming along this section that $\boldsymbol{W}$ is smooth and positive definite on the whole real axis $\mathbb{R}$. Some ideas about how to handle the case of finite endpoints of the support of $\boldsymbol{W}$ are briefly explained in the final Section 5 .

\subsection{The transformation}

As we have seen in Section 2.2, the recurrence relation (first identity in (2.28)) is a general fact intrinsic to the orthogonality of the polynomials. Simple differential relations are much more demanding, and we must impose at this stage further conditions on the orthogonality weight $\boldsymbol{W}$.

Our immediate goal is to obtain an invertible transformation $\boldsymbol{Y}^{n} \rightarrow \boldsymbol{X}^{n}$ such that $\boldsymbol{X}^{n}$ has a constant jump across $\mathbb{R}$. We will consider

$$
\boldsymbol{X}^{n}(z):=\boldsymbol{Y}^{n}(z) \boldsymbol{V}(z),
$$

where $\boldsymbol{V}$ is a matrix-valued function, analytic in $\mathbb{C} \backslash \mathbb{R}$ and continuous up to $\mathbb{R}$, and invertible for all $z \in \mathbb{C}$. Then the jump matrix for $\boldsymbol{X}^{n}$ on $\mathbb{R}$ is

$$
\boldsymbol{V}^{-1}\left(\begin{array}{cc}
\boldsymbol{I} & \boldsymbol{W} \\
\mathbf{0} & \boldsymbol{I}
\end{array}\right) \boldsymbol{V}
$$

Observe that this kind of transformations does not affect the first difference equation in the Lax pair (2.28), but constant jumps will allow us to use the strategy of variation of the problem along $z$. Going down the path of simplification, we can try a block-diagonal matrix

$$
\boldsymbol{V}(z)=\left(\begin{array}{c:c}
\boldsymbol{T}(z) & \mathbf{0} \\
\hdashline \mathbf{0} & \boldsymbol{T}^{-*}(z)
\end{array}\right),
$$


where $\boldsymbol{T}$ is an invertible $N \times N$ matrix function. Then (3.1) boils down to

$$
\left(\begin{array}{c:c}
\boldsymbol{I} & \boldsymbol{T}^{-1} \boldsymbol{W} \boldsymbol{T}^{-*} \\
\hdashline \mathbf{0} & \boldsymbol{I}
\end{array}\right)
$$

These preliminary considerations motivate to consider a factorization of the weight in the form

$$
\boldsymbol{W}(x)=\boldsymbol{T}(x) \boldsymbol{T}^{*}(x), \quad x \in \mathbb{R},
$$

where $\boldsymbol{T}$ is a smooth matrix-valued function on $\mathbb{R}$. Under our assumptions the existence of such a $\boldsymbol{T}$ is guaranteed, but not its uniqueness: performing its $R Q$ decomposition it can be written as

$$
\boldsymbol{T}(x)=\widehat{\boldsymbol{T}}(x) \boldsymbol{S}(x), \quad x \in \mathbb{R},
$$

where $\widehat{\boldsymbol{T}}(x)$ is an upper triangular matrix and $\boldsymbol{S}(x)$ is an arbitrary smooth and unitary matrix (for each $x \in \mathbb{R}$ ). This representation can go further taking into account that any unitary matrix can be written as $\boldsymbol{S}(x)=e^{\boldsymbol{Q}(x)}$, where $\boldsymbol{Q}(x)$ is an skew-Hermitian matrix function. Additionally, since any skew-Hermitian matrix is normal, it has a factorization $\boldsymbol{Q}(x)=i \boldsymbol{U}(x) \boldsymbol{D}(x) \boldsymbol{U}^{*}(x)$, where $\boldsymbol{U}$ is again unitary and $\boldsymbol{D}$ a diagonal matrix with real entries. Therefore $\boldsymbol{T}$ from (3.3) can be written as

$$
\boldsymbol{T}(x)=\widehat{\boldsymbol{T}}(x) \boldsymbol{U}(x) e^{i \boldsymbol{D}(x)} \boldsymbol{U}^{*}(x) .
$$

We narrow the choice of $\boldsymbol{T}$ (and $\boldsymbol{W}$ ) by imposing the additional constraint that there exists a matrix polynomial $\boldsymbol{G}$ such that in a neighborhood of the origin,

$$
\boldsymbol{T}^{\prime}(z)=\boldsymbol{G}(z) \boldsymbol{T}(z)
$$

As a consequence, (see the discussion in [39, Chapter 9] or [49, Chapter 1]), $\boldsymbol{T}$ has an analytic continuation to the whole plane as an entire and invertible matrix-valued function, and

$$
\boldsymbol{W}(z)=\boldsymbol{T}(z) \boldsymbol{T}^{*}(z)
$$

provides an analytic extension of $\boldsymbol{W}$ to the whole plane.

Following our previous discussion, we define the entire matrix-valued function

$$
\boldsymbol{V}(z)=\left(\begin{array}{c:c}
\boldsymbol{T}(z) & \mathbf{0} \\
\hdashline \mathbf{0} & \boldsymbol{T}^{-*}(z)
\end{array}\right) .
$$

Observe that $\boldsymbol{V}$ is invertible for all $z \in \mathbb{C}$. By differentiating the identity $\boldsymbol{T}^{-1} \boldsymbol{T}=\boldsymbol{I}$ and using (3.4) we conclude that $\left(\boldsymbol{T}^{-1}\right)^{\prime}=-\boldsymbol{T}^{-1} \boldsymbol{G}$, so that

$$
\boldsymbol{V}^{\prime}(z)=\left(\begin{array}{c:c}
\boldsymbol{G}(z) & \mathbf{0} \\
\hdashline \mathbf{0} & -\boldsymbol{G}^{*}(z)
\end{array}\right) \boldsymbol{V}(z) .
$$

If $\boldsymbol{Y}^{n}(z)$ is the solution of the original RHP, let us define

$$
\boldsymbol{X}^{n}(z)=\boldsymbol{Y}^{n}(z) \boldsymbol{V}(z)
$$

Then straightforward computations show that $\boldsymbol{X}^{n}(z)$ is analytic in $\mathbb{C} \backslash \mathbb{R}$, satisfies the jump condition

$$
\boldsymbol{X}_{+}^{n}(x)=\boldsymbol{X}_{-}^{n}(x)\left(\begin{array}{ccc}
\boldsymbol{I}_{N} & \boldsymbol{I}_{N} \\
\hdashline & 0 & \boldsymbol{I}_{N}
\end{array}\right), \quad x \in \mathbb{R},
$$


and for $m \in \mathbb{N}($ see $(2.3))$,

$$
\boldsymbol{X}^{n}(z)=\left(\boldsymbol{I}_{2 N}+\sum_{i=1}^{m} \frac{\boldsymbol{Y}_{i}^{n}}{z^{i}}+\mathcal{O}\left(1 / z^{m+1}\right)\right)\left(\begin{array}{c:c}
z^{n} \boldsymbol{I}_{N} & \mathbf{0} \\
\hdashline \mathbf{0} & z^{-n} \boldsymbol{I}_{N}
\end{array}\right) \boldsymbol{V}(z), \quad z \rightarrow \infty .
$$

Since $\boldsymbol{X}^{n}(z)$ is invertible in $\mathbb{C} \backslash \mathbb{R}$, we can consider the matrix function $\boldsymbol{F}_{n}(z)=\left[\frac{d}{d z} \boldsymbol{X}^{n}(z)\right] \boldsymbol{X}^{n}(z)^{-1}$. Again, $\boldsymbol{F}_{n}(z)$ is analytic in $\mathbb{C} \backslash \mathbb{R}$ and on the real line, $\left(\boldsymbol{F}_{n}\right)_{+}(x)=\left(\boldsymbol{F}_{n}\right)_{-}(x)$, which implies that it is an entire matrix function. From (2.3) and (3.7), for $z \rightarrow \infty$,

$$
\left[\frac{d}{d z} \boldsymbol{Y}^{n}(z)\right]\left[\boldsymbol{Y}^{n}(z)\right]^{-1}=\mathcal{O}\left(\frac{1}{z}\right)
$$

and combining it with (2.3), (2.5), (3.5) and (3.7), we get for $z \rightarrow \infty$,

$$
\begin{aligned}
& \boldsymbol{F}_{n}(z)=\left[\frac{d}{d z} \boldsymbol{X}^{n}(z)\right] \boldsymbol{X}^{n}(z)^{-1} \\
& =\left(\boldsymbol{I}_{2 N}+\sum_{i=1}^{m} \frac{\boldsymbol{Y}_{i}^{n}}{z^{i}}+\mathcal{O}\left(1 / z^{m+1}\right)\right)\left(\begin{array}{c:c}
\boldsymbol{G}(z) & \mathbf{0} \\
\hdashline \mathbf{0} & -\boldsymbol{G}^{*}(z)
\end{array}\right) \\
& \times\left(\boldsymbol{I}_{2 N}+\sum_{i=1}^{m} \frac{\tilde{\boldsymbol{Y}}_{i}^{n}}{z^{i}}+\mathcal{O}\left(1 / z^{m+1}\right)\right)+\mathcal{O}(1 / z) .
\end{aligned}
$$

By Liouville's theorem, the right hand side in (3.8) will coincide with its polynomial part in the expansion at infinity, and its degree is no greater than the degree of $\boldsymbol{G}$. To be more precise, if we assume that the degree of $\boldsymbol{G}$ is $m \in \mathbb{N}$ and denote

$$
\boldsymbol{G}(z)=\sum_{j=0}^{m} \boldsymbol{M}_{j} z^{j}, \quad \widetilde{\boldsymbol{M}}_{j}=\left(\begin{array}{c:c}
\boldsymbol{M}_{j} & \mathbf{0} \\
\hdashline \mathbf{0} & -\boldsymbol{M}_{j}^{*}
\end{array}\right),
$$

then after dropping the negative powers of $z$ in

$$
\begin{aligned}
\boldsymbol{F}_{n}(z)= & \left(\boldsymbol{I}_{2 N}+\sum_{i=1}^{m} \frac{\boldsymbol{Y}_{i}^{n}}{z^{i}}+\mathcal{O}\left(1 / z^{m+1}\right)\right) \\
& \times\left(\sum_{j=0}^{m} \widetilde{\boldsymbol{M}}_{j} z^{j}\right)\left(\boldsymbol{I}_{2 N}+\sum_{k=1}^{m} \frac{\widetilde{\boldsymbol{Y}}_{k}^{n}}{z^{k}}+\mathcal{O}\left(1 / z^{m+1}\right)\right)+\mathcal{O}(1 / z)
\end{aligned}
$$

we obtain that

$$
\boldsymbol{F}_{n}(z)=\sum_{k=0}^{m}\left(\sum_{j=k}^{m} \sum_{i=0}^{j-k} \boldsymbol{Y}_{i}^{n} \widetilde{\boldsymbol{M}}_{j} \tilde{\boldsymbol{Y}}_{j-i-k}^{n}\right) z^{k}, \quad \text { with } \quad \boldsymbol{Y}_{0}^{n}=\tilde{\boldsymbol{Y}}_{0}^{n}=\boldsymbol{I}_{2 N} .
$$

For instance, if $m=0$,

$$
\boldsymbol{F}_{n}(z)=\widetilde{\boldsymbol{M}}_{0},
$$

for $m=1$,

$$
\boldsymbol{F}_{n}(z)=\widetilde{\boldsymbol{M}}_{1} z+\widetilde{\boldsymbol{M}}_{0}+\boldsymbol{Y}_{1}^{n} \widetilde{\boldsymbol{M}}_{1}+\widetilde{\boldsymbol{M}}_{1} \widetilde{\boldsymbol{Y}}_{1}^{n}
$$

and for $m=2$,

$$
\begin{aligned}
\boldsymbol{F}_{n}(z)= & \widetilde{\boldsymbol{M}}_{2} z^{2}+\left(\widetilde{\boldsymbol{M}}_{1}+\boldsymbol{Y}_{1}^{n} \widetilde{\boldsymbol{M}}_{2}+\widetilde{\boldsymbol{M}}_{2} \widetilde{\boldsymbol{Y}}_{1}^{n}\right) z+\widetilde{\boldsymbol{M}}_{0}+\boldsymbol{Y}_{1}^{n} \widetilde{\boldsymbol{M}}_{1}+\widetilde{\boldsymbol{M}}_{1} \widetilde{\boldsymbol{Y}}_{1}^{n} \\
& +\boldsymbol{Y}_{2}^{n} \widetilde{\boldsymbol{M}}_{2}+\boldsymbol{Y}_{1}^{n} \widetilde{\boldsymbol{M}}_{2} \widetilde{\boldsymbol{Y}}_{1}^{n}+\widetilde{\boldsymbol{M}}_{2} \widetilde{\boldsymbol{Y}}_{2}^{n} .
\end{aligned}
$$

Finally, using the explicit expressions (2.22) and (2.23) in (3.9), we arrive at the following 
Theorem 3.1. Under assumptions (3.2) and (3.4), with $\boldsymbol{G}(z)=\sum_{j=0}^{m} \boldsymbol{M}_{j} z^{j} \in \mathbb{P}_{m}$, the matrix function $\boldsymbol{X}^{n}$ defined in (3.6) satisfies the following first-order differential equation with polynomial coefficients:

$$
\frac{d}{d z} \boldsymbol{X}^{n}(z)=\boldsymbol{F}_{n}(z ; \boldsymbol{G}) \boldsymbol{X}^{n}(z)
$$

where

$$
\boldsymbol{F}_{n}(z ; \boldsymbol{G})=\left(\begin{array}{c:c}
-\mathcal{B}_{n}(z ; \boldsymbol{G}) & -\frac{1}{2 \pi i} \boldsymbol{\gamma}_{n}^{-1} \mathcal{A}_{n}(z ; \boldsymbol{G}) \\
\hdashline 2 \pi i \mathcal{A}_{n-1}(z ; \boldsymbol{G}) \boldsymbol{\gamma}_{n-1} & \mathcal{B}_{n}^{*}(z ; \boldsymbol{G})
\end{array}\right),
$$

$\mathcal{A}_{n}$ and $\mathcal{B}_{n}$ are matrix polynomials,

$$
\begin{aligned}
& \mathcal{A}_{n}(z ; \boldsymbol{G})=-\boldsymbol{\gamma}_{n}\left(\sum_{j=0}^{m-1} \boldsymbol{b}_{n, n+m-j-1} \boldsymbol{\Delta}_{j, n}^{*}(z)+\boldsymbol{\Delta}_{j, n}(z) \boldsymbol{b}_{n, n+m-j-1}^{*}\right) \\
& \mathcal{B}_{n}(z ; \boldsymbol{G})=-\left(\sum_{j=0}^{m} \boldsymbol{\Delta}_{j, n}(z) \boldsymbol{b}_{n-1, n+m-j-1}^{*}+\boldsymbol{b}_{n, n+m-j-2} \boldsymbol{\Delta}_{j, n-1}^{*}(z)\right) \boldsymbol{\gamma}_{n-1},
\end{aligned}
$$

and the coefficients $\boldsymbol{\Delta}_{j, n}(z)$ are given by

$$
\boldsymbol{\Delta}_{j, n}(z)=\sum_{k=0}^{j} \widehat{\boldsymbol{P}}_{n, k}(z) \boldsymbol{M}_{m-j+k}, \quad \widehat{\boldsymbol{P}}_{n, k}(z)=z^{k} \boldsymbol{I}+\boldsymbol{a}_{n, n-1} z^{k-1}+\cdots+\boldsymbol{a}_{n, n-k} .
$$

Moreover, $\boldsymbol{F}_{n}(\cdot ; \boldsymbol{G})$ is linear in $\boldsymbol{G}$ :

$$
\boldsymbol{F}_{n}\left(z ; \boldsymbol{G}_{1}+\boldsymbol{G}_{2}\right)=\boldsymbol{F}_{n}\left(z ; \boldsymbol{G}_{1}\right)+\boldsymbol{F}_{n}\left(z ; \boldsymbol{G}_{2}\right) .
$$

Remark 3.2. The coefficients $\boldsymbol{b}_{n, k}$ were introduced in (2.20) and discussed in Lemma 2.10. It is worth observing the similarity of this result with Theorem 2.17; in the present situation we can compute the differential equation for $\boldsymbol{X}^{n}$ directly in terms of the coefficients of the MOPRL $\widehat{\boldsymbol{P}}_{n}$ without considering integrals or studying the behavior at the endpoints. Finally, we have once again that

$$
\gamma_{n} \mathcal{A}_{n}^{*}(z ; \boldsymbol{G})=\mathcal{A}_{n}(z ; \boldsymbol{G}) \boldsymbol{\gamma}_{n}
$$

Again, for lowest degrees $m$ in Theorem 3.1 we can use (3.10), (3.11), (3.12), Lemma 2.10 and Corollary 2.11 in order to write the coefficient matrix (3.14) of the differential equation explicitly. For instance, for $m=0$,

$$
\boldsymbol{F}_{n}(z ; \boldsymbol{G})=\left(\begin{array}{c:c}
\boldsymbol{M}_{0} & \mathbf{0} \\
\hdashline \mathbf{0} & -\boldsymbol{M}_{0}^{*}
\end{array}\right) ;
$$

for $m=1$,

$$
\boldsymbol{F}_{n}(z ; \boldsymbol{G})=\left(\begin{array}{c:c}
\boldsymbol{G}(z)+\boldsymbol{a}_{n, n-1} \boldsymbol{M}_{1}-\boldsymbol{M}_{1} \boldsymbol{a}_{n, n-1} & \frac{1}{2 \pi i}\left(\boldsymbol{\gamma}_{n}^{-1} \boldsymbol{M}_{1}^{*}+\boldsymbol{M}_{1} \boldsymbol{\gamma}_{n}^{-1}\right) \\
\hdashline-2 \pi i\left(\boldsymbol{\gamma}_{n-1} \boldsymbol{M}_{1}+\boldsymbol{M}_{1}^{*} \boldsymbol{\gamma}_{n-1}\right) & -\boldsymbol{G}^{*}(z)+\boldsymbol{a}_{n, n-1}^{*} \boldsymbol{M}_{1}^{*}-\boldsymbol{M}_{1}^{*} \boldsymbol{a}_{n, n-1}^{*}
\end{array}\right),
$$

and for $m=2$, with the notation (3.14),

$$
\mathcal{B}_{n}(z ; \boldsymbol{G})=-\boldsymbol{G}(z)-\left(\boldsymbol{a}_{n, n-1} \boldsymbol{M}_{2}-\boldsymbol{M}_{2} \boldsymbol{a}_{n, n-1}\right) z+\boldsymbol{M}_{1} \boldsymbol{a}_{n, n-1}-\boldsymbol{a}_{n, n-1} \boldsymbol{M}_{1}-\boldsymbol{a}_{n, n-2} \boldsymbol{M}_{2}
$$




$$
\begin{aligned}
& -\boldsymbol{M}_{2}\left(\boldsymbol{a}_{n+1, n} \boldsymbol{a}_{n, n-1}+\boldsymbol{a}_{n+1, n-1}\right)+\boldsymbol{a}_{n, n-1} \boldsymbol{M}_{2} \boldsymbol{a}_{n, n-1}-\boldsymbol{\gamma}_{n}^{-1} \boldsymbol{M}_{2}^{*} \boldsymbol{\gamma}_{n-1}, \\
\mathcal{A}_{n}(z ; \boldsymbol{G})= & -\boldsymbol{M}_{2}^{*} z-\boldsymbol{M}_{1}^{*}+\boldsymbol{a}_{n+1, n}^{*} \boldsymbol{M}_{2}^{*}-\boldsymbol{M}_{2}^{*} \boldsymbol{a}_{n, n-1}^{*} \\
& -\boldsymbol{\gamma}_{n}\left(\boldsymbol{M}_{2} z+\boldsymbol{M}_{1}-\boldsymbol{M}_{2} \boldsymbol{a}_{n+1, n}+\boldsymbol{a}_{n, n-1} \boldsymbol{M}_{2}\right) \boldsymbol{\gamma}_{n}^{-1}
\end{aligned}
$$

Observe from Theorem 3.1 that $\mathcal{B}_{n}(z ; \boldsymbol{G})$ is a matrix polynomial of degree at most $m$ and $\mathcal{A}_{n}(z ; \boldsymbol{G})$ is a matrix polynomial of degree at most $m-1$.

Finally, it is important to emphasize that in many situations we can exploit the implications of the freedom in the factorization (3.3) on the differential equation (3.13). This freedom, as we will see in Proposition 3.9, appears only in the matrix setting.

Proposition 3.3. If under assumptions (3.2) and (3.4), with $\boldsymbol{G}$ a polynomial, there exists a non-trivial matrix-valued function $\boldsymbol{S}$, non-singular on $\mathbb{C}$, smooth and unitary on $\mathbb{R}$, such that

$$
\boldsymbol{H}(z)=\boldsymbol{T}(z) \boldsymbol{S}^{\prime}(z) \boldsymbol{S}^{*}(z) \boldsymbol{T}^{-1}(z)
$$

is also a polynomial, then $\widetilde{\boldsymbol{T}}=\boldsymbol{T} \boldsymbol{S}$ satisfies

$$
\boldsymbol{W}(x)=\widetilde{\boldsymbol{T}}(x) \widetilde{\boldsymbol{T}}^{*}(x), \quad x \in \mathbb{R}, \quad \widetilde{\boldsymbol{T}}^{\prime}(z)=\widetilde{\boldsymbol{G}}(z) \widetilde{\boldsymbol{T}}(z), \quad z \in \mathbb{C},
$$

with $\widetilde{\boldsymbol{G}}(z)=\boldsymbol{G}(z)+\boldsymbol{H}(z)$. Moreover, the matrix $\boldsymbol{X}^{n}$ defined in (3.6), satisfies along with (3.13)-(3.16) the following relation:

$$
\frac{d}{d z} \boldsymbol{X}^{n}(z)=\left(\boldsymbol{F}_{n}(z ; \boldsymbol{G})+\boldsymbol{F}_{n}(z ; \boldsymbol{H})\right) \boldsymbol{X}^{n}(z)-\boldsymbol{X}^{n}(z)\left(\begin{array}{c:c}
\boldsymbol{\chi}(z) & \mathbf{0} \\
\hdashline \mathbf{0} & -\boldsymbol{\chi}^{*}(z)
\end{array}\right),
$$

with $\chi(z)=\boldsymbol{S}^{\prime}(z) \boldsymbol{S}^{*}(z)$.

Remark 3.4. Equations (3.13) and (3.21) are not necessarily trivially related, and in principle we could combine them in order to establish new relations for $\boldsymbol{X}^{n}$, and thus, for $\boldsymbol{Y}^{n}$.

Proof. The key observation is the formula (3.17), so that the coefficients $\mathcal{A}_{n}$ and $\mathcal{B}_{n}$ in (3.13) depend linearly on $G$ :

$$
\mathcal{A}_{n}(\cdot ; \boldsymbol{G}+\boldsymbol{H})=\mathcal{A}_{n}(\cdot ; \boldsymbol{G})+\mathcal{A}_{n}(\cdot ; \boldsymbol{H}), \quad \mathcal{B}_{n}(\cdot ; \boldsymbol{G}+\boldsymbol{H})=\mathcal{B}_{n}(\cdot ; \boldsymbol{G})+\mathcal{B}_{n}(\cdot ; \boldsymbol{H}) .
$$

\subsection{Differential properties}

Taking into account the similarities between Theorems 2.17 and 3.1, we get immediately the analogue of Proposition 2.20 (the compatibility conditions):

Proposition 3.5. Under assumptions (3.2) and (3.4), with $\boldsymbol{G}(z)$ a polynomial, the coefficients of the differential equation (3.13) satisfy the following recurrence relations: for every $n \geq 0$,

$$
\boldsymbol{I}+\mathcal{B}_{n+1}(z ; \boldsymbol{G})\left(z \boldsymbol{I}-\boldsymbol{\alpha}_{n}\right)-\left(z \boldsymbol{I}-\boldsymbol{\alpha}_{n}\right) \mathcal{B}_{n}(z ; \boldsymbol{G})=\mathcal{A}_{n+1}^{*}(z ; \boldsymbol{G}) \boldsymbol{\beta}_{n+1}-\boldsymbol{\beta}_{n} \mathcal{A}_{n-1}^{*}(z ; \boldsymbol{G})
$$

and

$$
\mathcal{B}_{n+1}(z ; \boldsymbol{G})+\gamma_{n}^{-1} \mathcal{B}_{n}^{*}(z ; \boldsymbol{G}) \gamma_{n}=\left(z \boldsymbol{I}-\boldsymbol{\alpha}_{n}\right) \mathcal{A}_{n}^{*}(z ; \boldsymbol{G}),
$$

where $\boldsymbol{\alpha}_{n}$ and $\boldsymbol{\beta}_{n}$ are the coefficients of the three-term recurrence relation (2.30).

As before, the ladder operators (the most basic differential properties for MOPRL) can be easily obtained by analyzing the first block column of $\boldsymbol{X}^{n}$ in the differential equation (3.13): 
Proposition 3.6. Under assumptions (3.2) and (3.4), with $\boldsymbol{G}(z)$ a matrix polynomial, the monic MOPRL $\left(\widehat{\boldsymbol{P}}_{n}\right)_{n}$ satisfy the following difference-differential relations (lowering and raising operators, respectively):

$$
\widehat{\boldsymbol{P}}_{n}^{\prime}(z)+\widehat{\boldsymbol{P}}_{n}(z) \boldsymbol{G}(z)=-\mathcal{B}_{n}(z ; \boldsymbol{G}) \widehat{\boldsymbol{P}}_{n}(z)+\mathcal{A}_{n}^{*}(z ; \boldsymbol{G}) \boldsymbol{\beta}_{n} \widehat{\boldsymbol{P}}_{n-1}(z)
$$

and

$$
\widehat{\boldsymbol{P}}_{n}^{\prime}(z)+\widehat{\boldsymbol{P}}_{n}(z) \boldsymbol{G}(z)=\left(\mathcal{A}_{n}^{*}(z ; \boldsymbol{G})\left(z \boldsymbol{I}-\boldsymbol{\alpha}_{n}\right)-\mathcal{B}_{n}(z ; \boldsymbol{G})\right) \widehat{\boldsymbol{P}}_{n}(z)-\mathcal{A}_{n}^{*}(z ; \boldsymbol{G}) \widehat{\boldsymbol{P}}_{n+1}(z),
$$

where $\mathcal{A}_{n}$ and $\mathcal{B}_{n}$ are given by (3.15) and (3.16), respectively.

Proof. Block entry $(1,1)$ of (3.13) gives the lowering operator (3.25), using (2.31) and (3.18), while block entry $(2,1)$ of $(3.13)$ gives the raising operator (3.26) using (3.24) and (3.18).

Remark 3.7. Comparing with the results of Corollary 2.21, notice that these ladder operators contain a term with the MOPRL multiplied on the left.

In the situation described in Proposition 3.3, we can in principle exploit the non-uniqueness of the relations above in order to derive further relations for the MOPRL. The following result shows the possibility of the existence of 2 -terms recurrence relations for the family $\widehat{\boldsymbol{P}}_{n}$, a phenomenon that has not been reported before in the theory of MOPRL.

Corollary 3.8. Under conditions of Proposition 3.3, the family of monic MOPRL $\left(\widehat{\boldsymbol{P}}_{n}\right)_{n}$ satisfies, along with (3.25)-(3.26), the following relations:

$$
\widehat{\boldsymbol{P}}_{n}(z) \boldsymbol{H}(z)=-\mathcal{B}_{n}(z ; \boldsymbol{H}) \widehat{\boldsymbol{P}}_{n}(z)+\mathcal{A}_{n}^{*}(z ; \boldsymbol{H}) \boldsymbol{\beta}_{n} \widehat{\boldsymbol{P}}_{n-1}(z)
$$

and

$$
\widehat{\boldsymbol{P}}_{n}(z) \boldsymbol{H}(z)=\left(\mathcal{A}_{n}^{*}(z ; \boldsymbol{H})\left(z \boldsymbol{I}-\boldsymbol{\alpha}_{n}\right)-\mathcal{B}_{n}(z ; \boldsymbol{H})\right) \widehat{\boldsymbol{P}}_{n}(z)-\mathcal{A}_{n}^{*}(z ; \boldsymbol{H}) \widehat{\boldsymbol{P}}_{n+1}(z),
$$

where $\boldsymbol{H}$ is defined in (3.20).

In particular, provided $\mathcal{A}_{n}(z ; \boldsymbol{G})$ is invertible for all $z \in \mathbb{C}$,

$$
\begin{array}{r}
\widehat{\boldsymbol{P}}_{n}(z) \boldsymbol{H}(z)+\mathcal{B}_{n}(z ; \boldsymbol{H}) \widehat{\boldsymbol{P}}_{n}(z)-\mathcal{A}_{n}^{*}(z ; \boldsymbol{H}) \mathcal{A}_{n}^{-*}(z ; \boldsymbol{G}) \\
\times\left(\widehat{\boldsymbol{P}}_{n}^{\prime}(z)+\widehat{\boldsymbol{P}}_{n}(z) \boldsymbol{G}(z)+\mathcal{B}_{n}(z ; \boldsymbol{G}) \widehat{\boldsymbol{P}}_{n}(z)\right)=\mathbf{0} .
\end{array}
$$

Proof. To get (3.27), we subtract (3.13) and (3.21) and then evaluate the $(1,1)$ block entry. (3.28) is obtained from (3.27) and the three term recurrence relation. Finally, (3.29) is obtained by replacing (3.25) in (3.27).

Equations (3.27) and (3.28) are known as the 0-th order ladder operators, while (3.29) is a first order differential relation for the MOPRL. In some situations they yield trivial identities; this is always true in the scalar case, as the following proposition shows:

Proposition 3.9. Assume that under the conditions of Proposition $3.3, \chi(z)=\boldsymbol{S}^{\prime}(z) \boldsymbol{S}^{*}(z)=$ $i p(z) \boldsymbol{I}$, where $p$ is a scalar polynomial of degree $m$. Then

$$
\mathcal{A}_{n}(z ; \boldsymbol{H})=\mathbf{0}, \quad \text { and } \quad \mathcal{B}_{n}(z ; \boldsymbol{H})=-i p(z) \boldsymbol{I} .
$$

Proof. From (3.20), $\boldsymbol{H}=\boldsymbol{T} \boldsymbol{\chi} \boldsymbol{T}^{-1}$, and by linearity of $\mathcal{A}_{n}$ and $\mathcal{B}_{n}$ in $\boldsymbol{H}$ (see (3.22)), it is enough to prove the formulas above for monomials $z^{k} \boldsymbol{I}$. In this case, formulas (3.15) and (3.16) simplify considerably. Using Proposition 2.14 gives $\mathcal{A}_{n}(z ; \boldsymbol{H})=\mathbf{0}$ and $\mathcal{B}_{n}(z ; \boldsymbol{H})=-i z^{k} \boldsymbol{I}$. 
In the scalar case $(N=1)$ the only smooth and unitary function $s(x)$ on $\mathbb{R}$ has the form $s(z)=e^{i p(x)}$, with $p$ real-valued, and

$$
s^{\prime}(z) \overline{s(\bar{z})}=i p(z),
$$

so that the assumption that $p$ is a polynomial brings us to the situation described in Proposition 3.9. This explains why in the scalar setting we never get nontrivial 0 -th order ladder operators. In the scalar case one cannot have first order differential relations for the MOPRL, such as those given in (3.29). The general matrix case is much richer and complex, as will be illustrated with some examples in the next section.

We finish this section with a class of second-order differential equations satisfied by the $\operatorname{MOPRL}\left(\widehat{\boldsymbol{P}}_{n}\right)_{n}$. As a consequence of the Proposition 3.6 we have the following

Proposition 3.10. Under assumptions (3.2) and (3.4), with $\boldsymbol{G}(z)$ a polynomial, the MOPRL $\left(\widehat{\boldsymbol{P}}_{n}\right)_{n}$ satisfy the following second-order differential equation

$$
\widehat{\boldsymbol{P}}_{n}^{\prime \prime}+2 \widehat{\boldsymbol{P}}_{n}^{\prime} \boldsymbol{G}+\widehat{\boldsymbol{P}}_{n}\left(\boldsymbol{G}^{\prime}+\boldsymbol{G}^{2}\right)+\mathcal{M}_{n} \widehat{\boldsymbol{P}}_{n}^{\prime}+\mathcal{N}_{n} \widehat{\boldsymbol{P}}_{n}+\mathcal{M}_{n} \widehat{\boldsymbol{P}}_{n} \boldsymbol{G}=\mathbf{0},
$$

where $\mathcal{A}_{n}(z)=\mathcal{A}_{n}(z ; \boldsymbol{G}), \mathcal{B}_{n}(z)=\mathcal{B}_{n}(z ; \boldsymbol{G})$,

$$
\begin{aligned}
\mathcal{M}_{n}(z) & =\mathcal{M}_{n}(z ; \boldsymbol{G}) \\
& =-\left(\mathcal{A}_{n}^{*}(z)\right)^{\prime} \mathcal{A}_{n}^{-*}(z)+\mathcal{B}_{n}(z)-\mathcal{A}_{n}^{*}(z)\left(z \boldsymbol{I}-\boldsymbol{\alpha}_{n}\right)+\mathcal{A}_{n}^{*}(z) \mathcal{B}_{n+1}(z) \mathcal{A}_{n}^{-*}(z),
\end{aligned}
$$

and

$$
\mathcal{N}_{n}(z)=\mathcal{N}_{n}(z ; \boldsymbol{G})=\mathcal{M}_{n}(z) \mathcal{B}_{n}(z)-\mathcal{B}_{n}^{2}(z)+\mathcal{B}_{n}^{\prime}(z)+\mathcal{A}_{n}^{*}(z) \boldsymbol{\beta}_{n} \mathcal{A}_{n-1}^{*}(z),
$$

provided that the inverse of $\mathcal{A}_{n}(z ; \boldsymbol{G})$ exists for $z \in \mathbb{C}$. Here, again, -* denotes the conjugate transpose of the inverse.

Proof. Differentiate the lowering operator (3.25) and substitute the raising operator (3.26) evaluated at $n-1$.

Remark 3.11. Note that we can easily obtain another second-order differential operator satisfied by the orthogonal polynomials reversing the ladder operators. This new differential equation needs not be in principle the same as (3.30). Nevertheless, it is straightforward to see that both equations are equivalent using the compatibility conditions (3.23) and (3.24).

The equation (3.30) does not have the form of the right hand side differential operator considered for instance in [23], due to the terms $\mathcal{M}_{n} \widehat{\boldsymbol{P}}_{n}^{\prime}, \mathcal{N}_{n} \widehat{\boldsymbol{P}}_{n}$ and $\mathcal{M}_{n} \widehat{\boldsymbol{P}}_{n} \boldsymbol{G}$. In some cases, under additional assumptions on the weight, (3.30) can be reduced further, as we will see in the following section.

\section{Illustrative examples}

In this section we study a number of examples of weights $\boldsymbol{W}$ which are smooth and non-vanishing on the whole real line; this assumption simplifies the Riemann-Hilbert formulation because in this case one does not consider the local conditions (Y4) (see Section 2.1). Additionally, without loss of generality, we take $\boldsymbol{W}(0)=\boldsymbol{I}$.

For convenience, we consider the weights of the form

$$
\boldsymbol{W}(x)=e^{-2 q(x)} \boldsymbol{U}(x) \boldsymbol{U}^{*}(x), \quad x \in \mathbb{R},
$$


where $q$ is a scalar real-valued function, so that with the notation (3.2) and (3.4), we may take

$$
\boldsymbol{T}(x)=e^{-q(x)} \boldsymbol{U}(x) \quad \text { and } \quad \boldsymbol{G}(x)=-q^{\prime}(x) \boldsymbol{I}+\boldsymbol{U}^{\prime}(x) \boldsymbol{U}^{-1}(x) .
$$

We are interested in the case when $\boldsymbol{G}$ is a matrix polynomial. Hence, keeping up with previous hypotheses, we will assume that $q$ is a (scalar) polynomial of even degree with real coefficients and a positive leading coefficient, and that $\boldsymbol{U}^{\prime}(x) \boldsymbol{U}^{-1}(x)$ is a matrix polynomial.

Since our main goal here is to generate a set of examples (some new, some already well known), we restrict the degree of $q$ to either 2 (the Hermite case) or 4 (the Freud case), and the degree of $\boldsymbol{U}^{\prime}(x) \boldsymbol{U}^{-1}(x)$ to at most 1 . We start by considering the case when $\boldsymbol{U}^{\prime}(x) \boldsymbol{U}^{-1}(x)$ is a monomial, i.e. either $\boldsymbol{U}^{\prime}(x) \boldsymbol{U}^{-1}(x)=\boldsymbol{A}$ or $\boldsymbol{U}^{\prime}(x) \boldsymbol{U}^{-1}(x)=2 \boldsymbol{B} x$ for constant matrices $\boldsymbol{A}, \boldsymbol{B} \in \mathbb{C}^{N \times N}$. Taking into account the linearity (3.22) of the coefficients $\mathcal{A}_{n}$ and $\mathcal{B}_{n}$ in (3.15), (3.16), we can obtain the differential equation (3.13) for the general case of $\boldsymbol{U}^{\prime}(x) \boldsymbol{U}^{-1}(x)=\boldsymbol{A}+2 \boldsymbol{B} x$. However, finding the corresponding orthogonality weight is more involved: when $\boldsymbol{A}$ and $\boldsymbol{B}$ do not commute, solving $\boldsymbol{U}^{\prime}(x) \boldsymbol{U}^{-1}(x)=\boldsymbol{A}+2 \boldsymbol{B} x$ is not straightforward. In Section 4.1.3 we will discuss some examples, which yield explicit expressions for $\boldsymbol{U}(x)$, related to this case.

Recently an example has been found in [3] where a weight matrix supported in the real line is explicitly given (but not of the type (4.1)), when $\boldsymbol{G}(x)$ is a matrix polynomial of degree $N$ with in general non-commuting coefficients.

\subsection{The Hermite case}

For $q(x)=x^{2} / 2$, let us consider two cases, first $\boldsymbol{U}^{\prime}(x) \boldsymbol{U}^{-1}(x)=\boldsymbol{A}$ and then $\boldsymbol{U}^{\prime}(x) \boldsymbol{U}^{-1}(x)=$ $2 \boldsymbol{B} x$. We end up this Section by discussing briefly the case of $\boldsymbol{U}^{\prime}(x) \boldsymbol{U}^{-1}(x)=\boldsymbol{A}+2 \boldsymbol{B} x$.

\subsection{1 $\quad U^{\prime}(x) U^{-1}(x)=A$}

The differential equation $\boldsymbol{U}^{\prime}(x) \boldsymbol{U}^{-1}(x)=\boldsymbol{A}$, so that $\boldsymbol{U}(x)=e^{\boldsymbol{A} x}, \boldsymbol{T}(x)=e^{-x^{2} / 2} e^{\boldsymbol{A} x}$, and the weight matrix (4.1) is given by

$$
\boldsymbol{W}(x)=e^{-x^{2}} e^{\boldsymbol{A} x} e^{\boldsymbol{A}^{*} x}, \quad \boldsymbol{A} \in \mathbb{C}^{N \times N}, \quad x \in \mathbb{R} .
$$

The matrix $\boldsymbol{G}$ has the form

$$
\boldsymbol{G}(x)=-x \boldsymbol{I}+\boldsymbol{A},
$$

and according to (3.19),

$$
\mathcal{A}_{n}(x ; \boldsymbol{G})=2 \boldsymbol{I}, \quad \mathcal{B}_{n}(x ; \boldsymbol{G})=-\boldsymbol{G}(x) .
$$

The compatibility conditions of Proposition 3.5 yield

$$
2\left(\boldsymbol{\beta}_{n+1}-\boldsymbol{\beta}_{n}\right)=\boldsymbol{I}+\boldsymbol{A} \boldsymbol{\alpha}_{n}-\boldsymbol{\alpha}_{n} \boldsymbol{A},
$$

and

$$
\boldsymbol{\alpha}_{n}=\frac{1}{2}\left(\boldsymbol{A}+\boldsymbol{\gamma}_{n}^{-1} \boldsymbol{A}^{*} \boldsymbol{\gamma}_{n}\right)
$$

The lowering and raising operators from Proposition 3.6 are reduced now to

$$
\widehat{\boldsymbol{P}}_{n}^{\prime}(x)+\widehat{\boldsymbol{P}}_{n}(x) \boldsymbol{A}-\boldsymbol{A} \widehat{\boldsymbol{P}}_{n}(x)=2 \boldsymbol{\beta}_{n} \widehat{\boldsymbol{P}}_{n-1}(x),
$$

and

$$
-\widehat{\boldsymbol{P}}_{n}^{\prime}(x)+2 x \widehat{\boldsymbol{P}}_{n}(x)+\boldsymbol{A} \widehat{\boldsymbol{P}}_{n}(x)-\widehat{\boldsymbol{P}}_{n}(x) \boldsymbol{A}-2 \boldsymbol{\alpha}_{n} \widehat{\boldsymbol{P}}_{n}(x)=2 \widehat{\boldsymbol{P}}_{n+1}(x) .
$$


Summing up the telescopic relation in (4.4) (or comparing the $\mathcal{O}\left(x^{n-1}\right)$ term in (4.6)) gives

$$
\boldsymbol{\beta}_{n}=\frac{1}{2}\left(n \boldsymbol{I}+\boldsymbol{a}_{n, n-1} \boldsymbol{A}-\boldsymbol{A} \boldsymbol{a}_{n, n-1}\right) .
$$

With the notation of Proposition 3.10,

$$
\mathcal{M}_{n}(x)=2\left(\boldsymbol{\alpha}_{n}-\boldsymbol{A}\right), \quad \mathcal{N}_{n}(x)=-2\left(\boldsymbol{\alpha}_{n}-\boldsymbol{A}\right) \boldsymbol{G}(x)-\boldsymbol{G}^{2}(x)+\boldsymbol{I}+4 \boldsymbol{\beta}_{n},
$$

so that the differential equation (3.30) for the monic polynomials $\widehat{\boldsymbol{P}}_{n}$, orthogonal with respect to the weight (4.3), boils down to

$$
\begin{aligned}
\widehat{\boldsymbol{P}}_{n}^{\prime \prime}(x) & +2 \widehat{\boldsymbol{P}}_{n}^{\prime}(x)(\boldsymbol{A}-x \boldsymbol{I})+\widehat{\boldsymbol{P}}_{n}(x)\left(\boldsymbol{A}^{2}-2 x \boldsymbol{A}\right) \\
= & \left(-2 x \boldsymbol{A}+\boldsymbol{A}^{2}-4 \boldsymbol{\beta}_{n}\right) \widehat{\boldsymbol{P}}_{n}(x)+2\left(\boldsymbol{A}-\boldsymbol{\alpha}_{n}\right)\left(\widehat{\boldsymbol{P}}_{n}^{\prime}(x)+\widehat{\boldsymbol{P}}_{n}(x) \boldsymbol{A}-\boldsymbol{A} \widehat{\boldsymbol{P}}_{n}(x)\right) .
\end{aligned}
$$

These formulas hold for any constant matrix $\boldsymbol{A}$, and we cannot expect important simplifications without narrowing the class of the weights further. This can be done assuming in addition that the hypotheses of Proposition 3.3 hold. This, as it was shown in [23, 24], imposes additional constraints on the weight $\boldsymbol{W}$. Since the construction is described in detail in $[23,24]$, the exposition in this part will be rather sketchy.

The matrix $\boldsymbol{H}$ from (3.20), restricted to $\mathbb{R}$, can be written as

$$
\begin{aligned}
\boldsymbol{H}(x) & =\boldsymbol{T}(x) \boldsymbol{\chi} \boldsymbol{T}^{-1}(x)=e^{\boldsymbol{A} x} \boldsymbol{\chi} e^{-\boldsymbol{A} x} \\
& =\boldsymbol{\chi}+\operatorname{ad}_{\boldsymbol{A}}(\boldsymbol{\chi}) x+\operatorname{ad}_{\boldsymbol{A}}^{2}(\boldsymbol{\chi}) \frac{x^{2}}{2}+\cdots=\sum_{k \geq 0} \operatorname{ad}_{\boldsymbol{A}}^{k}(\boldsymbol{\chi}) \frac{x^{k}}{k !}
\end{aligned}
$$

Here $\boldsymbol{\chi}(x)=\boldsymbol{S}^{\prime}(x) \boldsymbol{S}^{*}(x)$ is skew-Hermitian on $\mathbb{R}, \operatorname{ad}_{\boldsymbol{A}}$ is the commutator given by $\operatorname{ad}_{\boldsymbol{A}}(\boldsymbol{\chi})=$ $\boldsymbol{A} \boldsymbol{\chi}-\boldsymbol{\chi} \boldsymbol{A}$, and we define recursively

$$
\operatorname{ad}_{\boldsymbol{A}}^{0}(\chi)=\chi, \quad \operatorname{ad}_{\boldsymbol{A}}^{n+1}(\chi)=\operatorname{ad}_{\boldsymbol{A}}\left(\operatorname{ad}_{\boldsymbol{A}}^{n}(\chi)\right) \quad \text { for } \quad n \geq 1
$$

The simplest situation obtains when the right hand side in (4.9) is constant; as it was shown in Lemma 2.4 of [24], this assumption yields $\boldsymbol{\chi}=i a \boldsymbol{I}$ for certain $a \in \mathbb{R}$ (which is the case discussed in Proposition 3.9), when there are no new ladder operators. Consequently, for the first non-trivial situation we must assume that (4.9) is a matrix polynomial of degree at least one. We consider two situations that yield degree exactly one (see [23, 24] for motivations and further details).

Let us define a nilpotent matrix of the form

$$
\boldsymbol{L}=\sum_{k=1}^{N-1} \nu_{k} \boldsymbol{E}_{k, k+1}, \quad \nu_{k} \in \mathbb{C} \backslash\{0\},
$$

where $\boldsymbol{E}_{i j}$ is a matrix with 1 at entry $(i, j)$ and 0 elsewhere, and a diagonal matrix

$$
\boldsymbol{J}=\sum_{k=1}^{N}(N-k) \boldsymbol{E}_{k, k} .
$$

For the first non-trivial example we assume that $\boldsymbol{A}=\boldsymbol{L}$ and $\boldsymbol{\chi}=i \boldsymbol{J}$, so that

$$
\operatorname{ad}_{\boldsymbol{A}}(\chi)=-\boldsymbol{A}
$$

and $\operatorname{ad}_{\boldsymbol{A}}^{2}(\boldsymbol{\chi})=\mathbf{0}$. Thus, $\boldsymbol{H}(x)=e^{\boldsymbol{A} x} \boldsymbol{\chi} e^{-\boldsymbol{A} x}=i(\boldsymbol{J}-\boldsymbol{A} x)$, and by (3.19),

$$
\mathcal{A}_{n}(x ; \boldsymbol{H})=i\left(-\boldsymbol{A}^{*}+\boldsymbol{\gamma}_{n} \boldsymbol{A} \boldsymbol{\gamma}_{n}^{-1}\right)=2 i\left(\boldsymbol{\alpha}_{n}^{*}-\boldsymbol{A}^{*}\right),
$$




$$
\mathcal{B}_{n}(x ; \boldsymbol{H})=i\left(\boldsymbol{A} x-\boldsymbol{J}+\boldsymbol{a}_{n, n-1} \boldsymbol{A}-\boldsymbol{A} \boldsymbol{a}_{n, n-1}\right)=i\left(\boldsymbol{A} x-\boldsymbol{J}+2 \boldsymbol{\beta}_{n}-n \boldsymbol{I}\right) .
$$

From the compatibility conditions of Proposition 3.5 we get

$$
\begin{aligned}
& \boldsymbol{J} \boldsymbol{\alpha}_{n}-\boldsymbol{\alpha}_{n} \boldsymbol{J}+\boldsymbol{\alpha}_{n}=\boldsymbol{A}+\frac{1}{2}\left(\boldsymbol{A}^{2} \boldsymbol{\alpha}_{n}-\boldsymbol{\alpha}_{n} \boldsymbol{A}^{2}\right) \quad \text { and } \\
& \boldsymbol{J}-\boldsymbol{\gamma}_{n}^{-1} \boldsymbol{J} \boldsymbol{\gamma}_{n}=\boldsymbol{A} \boldsymbol{\alpha}_{n}+\boldsymbol{\alpha}_{n} \boldsymbol{A}-2 \boldsymbol{\alpha}_{n}^{2},
\end{aligned}
$$

where we have used relations (4.4), (4.5), (4.7) and

$$
\left(\boldsymbol{A}-2 \boldsymbol{\alpha}_{n}\right) \boldsymbol{\beta}_{n}=\boldsymbol{\beta}_{n}\left(\boldsymbol{A}-2 \boldsymbol{\alpha}_{n-1}\right),
$$

which can be easily proved using (2.31) and (4.5).

Thus, from Corollary 3.8, (4.4) and (4.13), the lowering and raising operators (of the 0-th order) are

$$
\begin{aligned}
& \widehat{\boldsymbol{P}}_{n}(x) \boldsymbol{J}-\boldsymbol{J} \widehat{\boldsymbol{P}}_{n}(x)-x\left(\widehat{\boldsymbol{P}}_{n}(x) \boldsymbol{A}-\boldsymbol{A} \widehat{\boldsymbol{P}}_{n}(x)\right)+2 \boldsymbol{\beta}_{n} \widehat{\boldsymbol{P}}_{n}(x)-n \widehat{\boldsymbol{P}}_{n}(x) \\
& =2\left(\boldsymbol{A}-\boldsymbol{\alpha}_{n}\right) \boldsymbol{\beta}_{n} \widehat{\boldsymbol{P}}_{n-1}(x),
\end{aligned}
$$

and

$$
\begin{aligned}
& \widehat{\boldsymbol{P}}_{n}(x)(\boldsymbol{J}-x \boldsymbol{A})-\boldsymbol{\gamma}_{n}^{-1}\left(\boldsymbol{J}-x \boldsymbol{A}^{*}\right) \boldsymbol{\gamma}_{n} \widehat{\boldsymbol{P}}_{n}(x)+2 \boldsymbol{\beta}_{n+1} \widehat{\boldsymbol{P}}_{n}(x)-(n+1) \widehat{\boldsymbol{P}}_{n}(x) \\
& \quad=2\left(\boldsymbol{\alpha}_{n}-\boldsymbol{A}\right) \widehat{\boldsymbol{P}}_{n+1}(x),
\end{aligned}
$$

respectively, which yields by (3.29) the first order relation

$$
\begin{aligned}
\left(\boldsymbol{A}-\boldsymbol{\alpha}_{n}\right) \widehat{\boldsymbol{P}}_{n}^{\prime}(x)+\left(\boldsymbol{A}-\boldsymbol{\alpha}_{n}+x \boldsymbol{I}\right)\left(\widehat{\boldsymbol{P}}_{n}(x) \boldsymbol{A}-\boldsymbol{A} \widehat{\boldsymbol{P}}_{n}(x)\right)-2 \boldsymbol{\beta}_{n} \widehat{\boldsymbol{P}}_{n}(x) & \\
= & \widehat{\boldsymbol{P}}_{n}(x) \boldsymbol{J}-\boldsymbol{J} \widehat{\boldsymbol{P}}_{n}(x)-n \widehat{\boldsymbol{P}}_{n}(x) .
\end{aligned}
$$

These identities hold in addition to the second order equation obtained in (4.8), valid as we recall, for any $\boldsymbol{A}$. As far as we are aware of, these relations are new. We use them to simplify the differential equation remarkably: multiplying (4.14) by 2 and plugging it into (4.8) gives us for $\boldsymbol{A}=\boldsymbol{L}$,

$$
\widehat{\boldsymbol{P}}_{n}^{\prime \prime}(x)+2 \widehat{\boldsymbol{P}}_{n}^{\prime}(x)(\boldsymbol{A}-x \boldsymbol{I})+\widehat{\boldsymbol{P}}_{n}(x)\left(\boldsymbol{A}^{2}-2 \boldsymbol{J}\right)=\left(-2 n \boldsymbol{I}+\boldsymbol{A}^{2}-2 \boldsymbol{J}\right) \widehat{\boldsymbol{P}}_{n}(x),
$$

which is a linear second-order differential equation with coefficients in the right hand side independent on $n$ (a.k.a. Sturm-Liouville equation with polynomial coefficients), studied by Durán and Grünbaum in [23].

In the second case we take

$$
\boldsymbol{A}=\boldsymbol{L}(\boldsymbol{I}+\boldsymbol{L})^{-1}=\sum_{j=1}^{N-1}(-1)^{j-1} \boldsymbol{L}^{j}, \quad \chi=i \boldsymbol{J},
$$

where $\boldsymbol{L}$ is given in (4.10) and $\boldsymbol{J}$ in (4.11). Consequently,

$$
\operatorname{ad}_{\boldsymbol{A}}(\boldsymbol{\chi})=-\boldsymbol{A}+\boldsymbol{A}^{2}, \quad \operatorname{ad}_{\boldsymbol{A}}^{2}(\boldsymbol{\chi})=\mathbf{0},
$$

and

$$
\boldsymbol{H}(x)=e^{\boldsymbol{A} x} \boldsymbol{\chi} e^{-\boldsymbol{A} x}=i\left(\boldsymbol{J}-\left(\boldsymbol{A}-\boldsymbol{A}^{2}\right) x\right) .
$$

In this case,

$$
\mathcal{A}_{n}(x ; \boldsymbol{H})=i\left(-\boldsymbol{A}^{*}+\left(\boldsymbol{A}^{*}\right)^{2}+\boldsymbol{\gamma}_{n}\left(\boldsymbol{A}-\boldsymbol{A}^{2}\right) \boldsymbol{\gamma}_{n}^{-1}\right)
$$




$$
=2 i\left(\boldsymbol{\alpha}_{n}^{*}-\boldsymbol{A}^{*}-\left(\boldsymbol{\alpha}_{n}^{*}-\boldsymbol{A}^{*}\right) \boldsymbol{\alpha}_{n}^{*}-\boldsymbol{\alpha}_{n}^{*}\left(\boldsymbol{\alpha}_{n}^{*}-\boldsymbol{A}^{*}\right)\right)
$$

and

$$
\begin{aligned}
\mathcal{B}_{n}(x ; \boldsymbol{H}) & =i\left(\left(\boldsymbol{A}-\boldsymbol{A}^{2}\right) x-\boldsymbol{J}+\boldsymbol{a}_{n, n-1}\left(\boldsymbol{A}-\boldsymbol{A}^{2}\right)-\left(\boldsymbol{A}-\boldsymbol{A}^{2}\right) \boldsymbol{a}_{n, n-1}\right) \\
& =i\left(\left(\boldsymbol{A}-\boldsymbol{A}^{2}\right) x-\boldsymbol{J}+2 \boldsymbol{\beta}_{n}-n \boldsymbol{I}-2\left(\boldsymbol{A} \boldsymbol{\beta}_{n}+\boldsymbol{\beta}_{n} \boldsymbol{A}\right)+2 n \boldsymbol{A}\right) .
\end{aligned}
$$

As to new results, we get the lowering operator,

$$
\begin{aligned}
\widehat{\boldsymbol{P}}_{n}(x) & \left(\boldsymbol{J}-x\left(\boldsymbol{A}-\boldsymbol{A}^{2}\right)\right)-\left(\boldsymbol{J}-x\left(\boldsymbol{A}-\boldsymbol{A}^{2}\right)\right) \widehat{\boldsymbol{P}}_{n}(x) \\
= & \left(-2 \boldsymbol{\beta}_{n}+n(\boldsymbol{I}-2 \boldsymbol{A})+2\left(\boldsymbol{A} \boldsymbol{\beta}_{n}+\boldsymbol{\beta}_{n} \boldsymbol{A}\right)\right) \widehat{\boldsymbol{P}}_{n}(x) \\
& -2\left(\boldsymbol{\alpha}_{n}-\boldsymbol{A}-\left(\boldsymbol{\alpha}_{n}-\boldsymbol{A}\right) \boldsymbol{\alpha}_{n}-\boldsymbol{\alpha}_{n}\left(\boldsymbol{\alpha}_{n}-\boldsymbol{A}\right)\right) \boldsymbol{\beta}_{n} \widehat{\boldsymbol{P}}_{n-1}(x),
\end{aligned}
$$

the raising operator

$$
\begin{aligned}
\widehat{\boldsymbol{P}}_{n}(x) & \left(\boldsymbol{J}-x\left(\boldsymbol{A}-\boldsymbol{A}^{2}\right)\right)-\left(\boldsymbol{J}-x\left(\boldsymbol{A}-\boldsymbol{A}^{2}\right)\right) \widehat{\boldsymbol{P}}_{n}(x) \\
= & \left(-2 \boldsymbol{\beta}_{n}+n(\boldsymbol{I}-2 \boldsymbol{A})+2\left(\boldsymbol{A} \boldsymbol{\beta}_{n}+\boldsymbol{\beta}_{n} \boldsymbol{A}\right)\right) \widehat{\boldsymbol{P}}_{n}(x) \\
& -2\left(\boldsymbol{\alpha}_{n}-\boldsymbol{A}-\left(\boldsymbol{\alpha}_{n}-\boldsymbol{A}\right) \boldsymbol{\alpha}_{n}-\boldsymbol{\alpha}_{n}\left(\boldsymbol{\alpha}_{n}-\boldsymbol{A}\right)\right)\left(\left(x \boldsymbol{I}-\boldsymbol{\alpha}_{n}\right) \widehat{\boldsymbol{P}}_{n}(x)-\widehat{\boldsymbol{P}}_{n+1}(x)\right),
\end{aligned}
$$

and the first-order differential equation (3.29):

$$
\begin{aligned}
\left(\boldsymbol{A}-\boldsymbol{\alpha}_{n}-\left(\boldsymbol{A}-\boldsymbol{\alpha}_{n}\right) \boldsymbol{\alpha}_{n}-\boldsymbol{\alpha}_{n}\left(\boldsymbol{A}-\boldsymbol{\alpha}_{n}\right)\right) \widehat{\boldsymbol{P}}_{n}^{\prime}(x) \\
=\widehat{\boldsymbol{P}}_{n}(x) \boldsymbol{J}-\boldsymbol{J} \widehat{\boldsymbol{P}}_{n}(x)-x\left(\widehat{\boldsymbol{P}}_{n}(x) \boldsymbol{A}^{2}-\boldsymbol{A}^{2} \widehat{\boldsymbol{P}}_{n}(x)\right) \\
\quad+\left(x \boldsymbol{I}-\boldsymbol{A}+\boldsymbol{\alpha}_{n}+\left(\boldsymbol{A}-\boldsymbol{\alpha}_{n}\right) \boldsymbol{\alpha}_{n}+\boldsymbol{\alpha}_{n}\left(\boldsymbol{A}-\boldsymbol{\alpha}_{n}\right)\right)\left(\widehat{\boldsymbol{P}}_{n}(x) \boldsymbol{A}-\boldsymbol{A} \widehat{\boldsymbol{P}}_{n}(x)\right) \\
\quad+\left(2 \boldsymbol{\beta}_{n}+n(2 \boldsymbol{A}-\boldsymbol{I})-2\left(\boldsymbol{A} \boldsymbol{\beta}_{n}+\boldsymbol{\beta}_{n} \boldsymbol{A}\right)\right) \widehat{\boldsymbol{P}}_{n}(x) .
\end{aligned}
$$

Multiplying this equation by 2 and plugging it into the second-order differential equation (4.8) gives

$$
\begin{aligned}
\widehat{\boldsymbol{P}}_{n}^{\prime \prime}(x) & +2 \widehat{\boldsymbol{P}}_{n}^{\prime}(x)(\boldsymbol{A}-x \boldsymbol{I})+\widehat{\boldsymbol{P}}_{n}(x)\left(\boldsymbol{A}^{2}-2 x \boldsymbol{A}^{2}-2 \boldsymbol{J}\right)=\left(\boldsymbol{A}^{2}-2 x \boldsymbol{A}^{2}-2 \boldsymbol{J}\right) \widehat{\boldsymbol{P}}_{n}(x) \\
& +\left(2 n(2 \boldsymbol{A}-\boldsymbol{I})-4\left(\boldsymbol{A} \boldsymbol{\beta}_{n}+\boldsymbol{\beta}_{n} \boldsymbol{A}\right)+2\left(\left(\boldsymbol{\alpha}_{n}-\boldsymbol{A}\right) \boldsymbol{\alpha}_{n}+\boldsymbol{\alpha}_{n}\left(\boldsymbol{\alpha}_{n}-\boldsymbol{A}\right)\right) \boldsymbol{A}\right) \widehat{\boldsymbol{P}}_{n}(x) \\
& -2\left(\left(\boldsymbol{\alpha}_{n}-\boldsymbol{A}\right) \boldsymbol{\alpha}_{n}+\boldsymbol{\alpha}_{n}\left(\boldsymbol{\alpha}_{n}-\boldsymbol{A}\right)\right)\left(\widehat{\boldsymbol{P}}_{n}^{\prime}(x)+\widehat{\boldsymbol{P}}_{n}(x) \boldsymbol{A}\right) .
\end{aligned}
$$

This differential equation is not of Sturm-Liouville type considered by Durán and Grünbaum in [23], but nevertheless we have been able to give a number of differential equations of first and second order satisfied by MOPRL with respect to a weight matrix that have not been considered up to this point.

\subsection{2 $U^{\prime}(x) U^{-1}(x)=2 B x$}

The weight matrix (4.1) is now given by

$$
\boldsymbol{W}(x)=e^{-x^{2}} e^{\boldsymbol{B} x^{2}} e^{\boldsymbol{B}^{*} x^{2}}, \quad \boldsymbol{B} \in \mathbb{C}^{N \times N}, \quad x \in \mathbb{R},
$$

where we assume that $\boldsymbol{B}$ is chosen such that all the moments exist. By (4.2),

$$
\boldsymbol{T}(x)=e^{-x^{2} / 2} e^{\boldsymbol{B} x^{2}} \quad \text { and } \quad \boldsymbol{G}(x)=(2 \boldsymbol{B}-\boldsymbol{I}) x .
$$

The weight matrix is an even matrix function, so that all moments of odd order vanish. As a consequence, $\boldsymbol{a}_{n, n-1}=\mathbf{0}$ and $\boldsymbol{\alpha}_{n}=\mathbf{0}$. Then

$$
\mathcal{A}_{n}(x ; \boldsymbol{G})=2\left(\boldsymbol{I}-\boldsymbol{B}^{*}-\boldsymbol{\gamma}_{n} \boldsymbol{B} \boldsymbol{\gamma}_{n}^{-1}\right), \quad \mathcal{B}_{n}(x ; \boldsymbol{G})=(\boldsymbol{I}-2 \boldsymbol{B}) x,
$$


and there will be only one compatibility condition:

$$
2\left(\boldsymbol{I}-\boldsymbol{B}-\boldsymbol{\gamma}_{n+1}^{-1} \boldsymbol{B}^{*} \boldsymbol{\gamma}_{n+1}\right) \boldsymbol{\beta}_{n+1}-2 \boldsymbol{\beta}_{n}\left(\boldsymbol{I}-\boldsymbol{B}-\boldsymbol{\gamma}_{n-1}^{-1} \boldsymbol{B}^{*} \boldsymbol{\gamma}_{n-1}\right)=\boldsymbol{I} .
$$

From (4.16) we get the explicit expression for $\boldsymbol{\beta}_{n}$ via

$$
2\left(\boldsymbol{I}-\boldsymbol{B}-\boldsymbol{\gamma}_{n}^{-1} \boldsymbol{B}^{*} \boldsymbol{\gamma}_{n}\right) \boldsymbol{\beta}_{n}=n \boldsymbol{I}+2\left(\boldsymbol{a}_{n, n-2} \boldsymbol{B}-\boldsymbol{B} \boldsymbol{a}_{n, n-2}\right) .
$$

The lowering and raising operators are

$$
\widehat{\boldsymbol{P}}_{n}^{\prime}(x)+2 x\left(\widehat{\boldsymbol{P}}_{n}(x) \boldsymbol{B}-\boldsymbol{B} \widehat{\boldsymbol{P}}_{n}(x)\right)=2\left(\boldsymbol{I}-\boldsymbol{B}-\boldsymbol{\gamma}_{n}^{-1} \boldsymbol{B}^{*} \boldsymbol{\gamma}_{n}\right) \boldsymbol{\beta}_{n} \widehat{\boldsymbol{P}}_{n-1}(x),
$$

and

$$
\widehat{\boldsymbol{P}}_{n}^{\prime}(x)+2 x\left(\widehat{\boldsymbol{P}}_{n}(x) \boldsymbol{B}-\boldsymbol{B} \widehat{\boldsymbol{P}}_{n}(x)\right)=2\left(\boldsymbol{I}-\boldsymbol{B}-\boldsymbol{\gamma}_{n}^{-1} \boldsymbol{B}^{*} \boldsymbol{\gamma}_{n}\right)\left(x \widehat{\boldsymbol{P}}_{n}(x)-\widehat{\boldsymbol{P}}_{n+1}(x)\right),
$$

respectively. This yields the following second-order differential equation

$$
\begin{gathered}
\widehat{\boldsymbol{P}}_{n}^{\prime \prime}(x)+2 x \widehat{\boldsymbol{P}}_{n}^{\prime}(x)(2 \boldsymbol{B}-\boldsymbol{I})+2 x\left(\boldsymbol{\gamma}_{n}^{-1} \boldsymbol{B}^{*} \boldsymbol{\gamma}_{n}-\boldsymbol{L}_{n}\right) \widehat{\boldsymbol{P}}_{n}^{\prime}(x)+4 x^{2}\left(\widehat{\boldsymbol{P}}_{n}(x) \boldsymbol{B}^{2}-\boldsymbol{B}^{2} \widehat{\boldsymbol{P}}_{n}(x)\right) \\
+4 \boldsymbol{K}_{n} \widehat{\boldsymbol{P}}_{n}(x)+\left(\left(2-4 x^{2}\right) \boldsymbol{I}+4 x^{2}\left(\boldsymbol{\gamma}_{n}^{-1} \boldsymbol{B}^{*} \boldsymbol{\gamma}_{n}-\boldsymbol{L}_{n}\right)\right)\left(\widehat{\boldsymbol{P}}_{n}(x) \boldsymbol{B}-\boldsymbol{B} \widehat{\boldsymbol{P}}_{n}(x)\right)=\mathbf{0},
\end{gathered}
$$

where

$$
\boldsymbol{L}_{n}=\left(\boldsymbol{I}-\boldsymbol{B}-\boldsymbol{\gamma}_{n}^{-1} \boldsymbol{B}^{*} \boldsymbol{\gamma}_{n}\right) \boldsymbol{B}\left(\boldsymbol{I}-\boldsymbol{B}-\boldsymbol{\gamma}_{n}^{-1} \boldsymbol{B}^{*} \boldsymbol{\gamma}_{n}\right)^{-1},
$$

and

$$
\boldsymbol{K}_{n}=\left(\boldsymbol{I}-\boldsymbol{B}-\boldsymbol{\gamma}_{n}^{-1} \boldsymbol{B}^{*} \boldsymbol{\gamma}_{n}\right) \boldsymbol{\beta}_{n}\left(\boldsymbol{I}-\boldsymbol{B}-\boldsymbol{\gamma}_{n-1}^{-1} \boldsymbol{B}^{*} \boldsymbol{\gamma}_{n-1}\right)
$$

These expressions are valid for any $\boldsymbol{B}$ (as long as all moments of the weight matrix exist). For further simplifications we can assume again that the hypotheses of Proposition 3.3 hold, and consider the cases given by the algebraic relations (4.12) and (4.15). For instance, when (4.15) holds, we obtain again a second-order differential equation with coefficients in the right hand side independent on $n$, studied by Durán and Grünbaum in [23]. The computations are similar and will be omitted for the sake of brevity.

\subsubsection{Other cases}

Assume now that we have a linear combination of the previous two cases, i.e.

$$
\boldsymbol{U}^{\prime}(x) \boldsymbol{U}^{-1}(x)=\boldsymbol{A}+2 \boldsymbol{B} x, \quad \boldsymbol{A}, \boldsymbol{B} \in \mathbb{C}^{N \times N}, \quad \text { with } \quad \boldsymbol{U}(0)=\boldsymbol{I},
$$

in which case the matrix $\boldsymbol{G}(x)$ is given by $\boldsymbol{G}(x)=\boldsymbol{A}+(2 \boldsymbol{B}-\boldsymbol{I}) x$. This is all that is needed to calculate the coefficients $\mathcal{A}_{n}(x ; \boldsymbol{G})$ and $\mathcal{B}_{n}(x ; \boldsymbol{G})$ and consequently the compatibility conditions, the ladder operators and the differential relations.

However, for the corresponding orthogonality weight we need to solve (4.17) explicitly, which may be non-trivial (unless $\boldsymbol{A}$ and $\boldsymbol{B}$ commute). In general, this solution can be given in terms of the time ordered exponential

$$
\boldsymbol{U}(x)=: e^{\int_{0}^{x}(\boldsymbol{A}+\boldsymbol{B} s) d s}:, \quad \boldsymbol{A}, \boldsymbol{B} \in \mathbb{C}^{N \times N}, \quad x \in \mathbb{R} .
$$

This is obtained by rewriting the differential equation as an integral one and "solving" it by iteration. In general, one cannot give an explicit expression for this infinite sum.

An example of explicitly solvable non-trivial equation (4.17) can be found in [22, Theorem 1.1], where non-commuting $\boldsymbol{A}$ and $\boldsymbol{B}$ are constructed (as a linear combination of the matrices $\boldsymbol{L}$ and $\boldsymbol{J}$ 
in (4.10) and (4.11), respectively) such that the corresponding $\boldsymbol{U}(x)=e^{\left(\boldsymbol{L}-v_{0} \boldsymbol{J}\right) x} e^{v_{0} \boldsymbol{J} x}$, with $v_{0}$ any real number. [22] contains also another example of a pair of non-commuting matrices $\boldsymbol{A}$ and $\boldsymbol{B}$ such that $\boldsymbol{U}(x)$ is a matrix polynomial of degree $N-1$, whose coefficients can be obtained recursively in terms of $\boldsymbol{A}$ and $\boldsymbol{B}$ (see Theorem A.1 therein).

An alternative way of generating examples is by considering weight matrices $\boldsymbol{W}$ of the form (4.1) with $\boldsymbol{U}(x) \in \mathbb{P}_{m}$ such that $\boldsymbol{U}(0)=\boldsymbol{I}$ and $\operatorname{det} \boldsymbol{U}(x)=1$ for $x \in \mathbb{R}$. If we denote

$$
\boldsymbol{U}(x)=\boldsymbol{I}+\sum_{k=1}^{m} \boldsymbol{A}_{k} x^{k}, \quad \boldsymbol{U}^{-1}(x)=\boldsymbol{I}+\sum_{k=1}^{m} \boldsymbol{B}_{k} x^{k},
$$

then the coefficients $\boldsymbol{A}_{k}$ and $\boldsymbol{B}_{k}$ are connected by simple algebraic relations. For instance,

$$
\boldsymbol{B}_{1}=-\boldsymbol{A}_{1}, \quad \boldsymbol{B}_{2}=-\boldsymbol{A}_{2}+\boldsymbol{A}_{1}^{2}, \quad \boldsymbol{B}_{3}=-\boldsymbol{A}_{3}+\boldsymbol{A}_{2} \boldsymbol{A}_{1}+\boldsymbol{A}_{1} \boldsymbol{A}_{2}-\boldsymbol{A}_{1}^{3} .
$$

In consequence,

$$
\begin{aligned}
& \boldsymbol{U}^{\prime}(x) \boldsymbol{U}^{-1}(x)=\boldsymbol{A}_{1}+\left(2 \boldsymbol{A}_{2}-\boldsymbol{A}_{1}^{2}\right) x+\left(3 \boldsymbol{A}_{3}-2 \boldsymbol{A}_{2} \boldsymbol{A}_{1}-\boldsymbol{A}_{1} \boldsymbol{A}_{2}+\boldsymbol{A}_{1}^{3}\right) x^{2} \\
& +\left(4 \boldsymbol{A}_{4}-3 \boldsymbol{A}_{3} \boldsymbol{A}_{1}-\boldsymbol{A}_{1} \boldsymbol{A}_{3}+2 \boldsymbol{A}_{2} \boldsymbol{A}_{1}^{2}+\boldsymbol{A}_{1}^{2} \boldsymbol{A}_{2}+\boldsymbol{A}_{1} \boldsymbol{A}_{2} \boldsymbol{A}_{1}-2 \boldsymbol{A}_{2}^{2}-\boldsymbol{A}_{1}^{4}\right) x^{3}+\cdots
\end{aligned}
$$

Let us discuss some cases, depending on the degree $m$ of the matrix polynomial $\boldsymbol{U}(x)$. In the first non-trivial example, when $m=1$, we have $\boldsymbol{U}(x)=\boldsymbol{I}+\boldsymbol{A}_{1} x$ and $\boldsymbol{U}^{-1}(x)=\boldsymbol{I}-\boldsymbol{A}_{1} x$ with $\boldsymbol{A}_{1}^{2}=\mathbf{0}$. Therefore, $\boldsymbol{U}^{\prime}(x) \boldsymbol{U}^{-1}(x)=\boldsymbol{A}_{1}$, which was considered already in Section 4.1.1.

Let $m=2$, so that $\boldsymbol{U}(x)=\boldsymbol{I}+\boldsymbol{A}_{1} x+\boldsymbol{A}_{2} x^{2}$ and $\boldsymbol{U}^{-1}(x)=\boldsymbol{I}-\boldsymbol{A}_{1} x+\left(\boldsymbol{A}_{1}^{2}-\boldsymbol{A}_{2}\right) x^{2}$. The condition $\boldsymbol{U}(x) \boldsymbol{U}^{-1}(x)=\boldsymbol{I}$ yields the following algebraic relations between the coefficients $\boldsymbol{A}_{1}$ and $\boldsymbol{A}_{2}$ :

$$
\boldsymbol{A}_{2} \boldsymbol{A}_{1}+\boldsymbol{A}_{1} \boldsymbol{A}_{2}=\boldsymbol{A}_{1}^{3}, \quad \boldsymbol{A}_{2}\left(-\boldsymbol{A}_{2}+\boldsymbol{A}_{1}^{2}\right)=\mathbf{0} .
$$

Algebraic manipulations of these two equations give additionally $\boldsymbol{A}_{2} \boldsymbol{A}_{1} \boldsymbol{A}_{2}=\mathbf{0}$ and $\boldsymbol{A}_{2} \boldsymbol{A}_{1}^{2}=$ $\boldsymbol{A}_{1}^{2} \boldsymbol{A}_{2}$. Therefore

$$
\boldsymbol{G}(x)=\boldsymbol{A}_{1}+\left(2 \boldsymbol{A}_{2}-\boldsymbol{A}_{1}^{2}-\boldsymbol{I}\right) x-\boldsymbol{A}_{2} \boldsymbol{A}_{1} x^{2} .
$$

The simplest case when $\boldsymbol{G}(x)$ is a matrix polynomial of degree one gives another algebraic equation, $\boldsymbol{A}_{2} \boldsymbol{A}_{1}=\mathbf{0}$. This immediately implies that $\boldsymbol{A}_{2}^{2}=\mathbf{0}$ and $\boldsymbol{A}_{1}^{4}=\mathbf{0}$, i.e. once again resulting in very strong algebraic conditions on the coefficients. In order to find an interesting example ( $\operatorname{when}^{\operatorname{ad}_{\boldsymbol{A}_{1}}}\left(\boldsymbol{A}_{2}\right) \neq \mathbf{0}$ ) we have to go at least to the dimension $N=4$; for instance,

$$
\begin{gathered}
\boldsymbol{A}_{1}=\left(\begin{array}{cccc}
0 & a_{12} & a_{13} & a_{14} \\
0 & 0 & a_{23} & a_{24} \\
0 & 0 & 0 & a_{34} \\
0 & 0 & 0 & 0
\end{array}\right), \quad \boldsymbol{A}_{2}=\left(\begin{array}{cccc}
0 & 0 & 0 & b_{14} \\
0 & 0 & 0 & a_{23} a_{34} \\
0 & 0 & 0 & 0 \\
0 & 0 & 0 & 0
\end{array}\right), \\
\operatorname{ad}_{\boldsymbol{A}_{1}}\left(\boldsymbol{A}_{2}\right)=\left(\begin{array}{cccc}
0 & 0 & 0 & a_{12} a_{23} a_{34} \\
0 & 0 & 0 & 0 \\
0 & 0 & 0 & 0 \\
0 & 0 & 0 & 0
\end{array}\right) .
\end{gathered}
$$

Considering higher degree examples will give more and more algebraic relations among the coefficients $\boldsymbol{A}_{k}$, which in general are difficult to solve.

In particular, in the examples that have appeared in the literature so far, $\boldsymbol{U}(x)$ is in general a matrix polynomial of degree depending on the size $N$. 


\subsection{The Freud case}

Now we have $q(x)=\frac{x^{4}}{2}$, so the weight matrix is given by

$$
\boldsymbol{W}(x)=e^{-x^{4}} \boldsymbol{U}(x) \boldsymbol{U}^{*}(x), \quad x \in \mathbb{R} .
$$

Again, we will consider two cases, first $\boldsymbol{U}^{\prime}(x) \boldsymbol{U}^{-1}(x)=\boldsymbol{A}$ and then $\boldsymbol{U}^{\prime}(x) \boldsymbol{U}^{-1}(x)=2 \boldsymbol{B} x$.

\subsection{1 $U^{\prime}(x) U^{-1}(x)=A$}

The weight matrix (4.1) in this case is given by

$$
\boldsymbol{W}(x)=e^{-x^{4}} e^{\boldsymbol{A} x} e^{\boldsymbol{A}^{*} x}, \quad \boldsymbol{A} \in \mathbb{C}^{N \times N}, \quad x \in \mathbb{R} .
$$

Then $\boldsymbol{T}(x)=e^{-x^{4} / 2} e^{\boldsymbol{A} x}, \boldsymbol{G}$ is given by

$$
\boldsymbol{G}(x)=-2 x^{3} \boldsymbol{I}+\boldsymbol{A} .
$$

Therefore using (3.15) and (3.16) we get

$$
\mathcal{A}_{n}(x ; \boldsymbol{G})=4\left(x^{2} \boldsymbol{I}+\boldsymbol{\alpha}_{n} x+\boldsymbol{\beta}_{n+1}+\boldsymbol{\beta}_{n}+\boldsymbol{\alpha}_{n}^{2}\right)^{*},
$$

and

$$
\mathcal{B}_{n}(x ; \boldsymbol{G})=2 x^{3} \boldsymbol{I}+4\left(\boldsymbol{\beta}_{n} x+\boldsymbol{\beta}_{n} \boldsymbol{\alpha}_{n-1}+\boldsymbol{\alpha}_{n} \boldsymbol{\beta}_{n}\right)-\boldsymbol{A} .
$$

These formulas are obtained by using the relation between the coefficients of the three-term recurrence equation (2.30) and the coefficients of the MOPRL. In this case the coefficients $\mathcal{A}_{n}(\cdot ; \boldsymbol{G})$ and $\mathcal{B}_{n}(\cdot ; \boldsymbol{G})$ are matrix polynomials of degree 2 and 3, respectively.

From Proposition 3.5 the compatibility conditions are

$$
\begin{gathered}
\boldsymbol{I}+\boldsymbol{A} \boldsymbol{\alpha}_{n}-\boldsymbol{\alpha}_{n} \boldsymbol{A}-4\left(\boldsymbol{\beta}_{n+1} \boldsymbol{\alpha}_{n}+\boldsymbol{\alpha}_{n+1} \boldsymbol{\beta}_{n+1}\right) \boldsymbol{\alpha}_{n}+4 \boldsymbol{\alpha}_{n}\left(\boldsymbol{\beta}_{n} \boldsymbol{\alpha}_{n-1}+\boldsymbol{\alpha}_{n} \boldsymbol{\beta}_{n}\right) \\
=4\left(\boldsymbol{\beta}_{n+2}+\boldsymbol{\beta}_{n+1}+\boldsymbol{\alpha}_{n+1}^{2}\right) \boldsymbol{\beta}_{n+1}-4 \boldsymbol{\beta}_{n}\left(\boldsymbol{\beta}_{n}+\boldsymbol{\beta}_{n-1}+\boldsymbol{\alpha}_{n-1}^{2}\right),
\end{gathered}
$$

and

$$
4\left(\left(\boldsymbol{\beta}_{n+1}+\boldsymbol{\beta}_{n}+\boldsymbol{\alpha}_{n}^{2}\right) \boldsymbol{\alpha}_{n}+\boldsymbol{\alpha}_{n}\left(\boldsymbol{\beta}_{n+1}+\boldsymbol{\beta}_{n}\right)+\boldsymbol{\alpha}_{n+1} \boldsymbol{\beta}_{n+1}+\boldsymbol{\beta}_{n} \boldsymbol{\alpha}_{n-1}\right)=\boldsymbol{A}+\boldsymbol{\gamma}_{n}^{-1} \boldsymbol{A}^{*} \boldsymbol{\gamma}_{n}
$$

The lowering operator is

$$
\begin{aligned}
\widehat{\boldsymbol{P}}_{n}^{\prime}(x) & +\widehat{\boldsymbol{P}}_{n}(x) \boldsymbol{A}-\boldsymbol{A} \widehat{\boldsymbol{P}}_{n}(x)=-4\left(\boldsymbol{\beta}_{n} x+\boldsymbol{\beta}_{n} \boldsymbol{\alpha}_{n-1}+\boldsymbol{\alpha}_{n} \boldsymbol{\beta}_{n}\right) \widehat{\boldsymbol{P}}_{n}(x) \\
& +4\left(x^{2} \boldsymbol{I}+\boldsymbol{\alpha}_{n} x+\boldsymbol{\beta}_{n+1}+\boldsymbol{\beta}_{n}+\boldsymbol{\alpha}_{n}^{2}\right) \boldsymbol{\beta}_{n} \widehat{\boldsymbol{P}}_{n-1}(x),
\end{aligned}
$$

while the raising operator is

$$
\begin{aligned}
\widehat{\boldsymbol{P}}_{n}^{\prime}(x) & +\widehat{\boldsymbol{P}}_{n}(x) \boldsymbol{A}-\boldsymbol{A} \widehat{\boldsymbol{P}}_{n}(x) \\
= & 4\left(x^{3} \boldsymbol{I}+\boldsymbol{\beta}_{n+1} x-\left(\boldsymbol{\beta}_{n+1}+\boldsymbol{\beta}_{n}+\boldsymbol{\alpha}_{n}^{2}\right) \boldsymbol{\alpha}_{n}-\boldsymbol{\beta}_{n} \boldsymbol{\alpha}_{n-1}-\boldsymbol{\alpha}_{n} \boldsymbol{\beta}_{n}\right) \widehat{\boldsymbol{P}}_{n}(x) \\
& -4\left(x^{2} \boldsymbol{I}+\boldsymbol{\alpha}_{n} x+\boldsymbol{\beta}_{n+1}+\boldsymbol{\beta}_{n}+\boldsymbol{\alpha}_{n}^{2}\right) \widehat{\boldsymbol{P}}_{n+1}(x) .
\end{aligned}
$$

From the $\mathcal{O}\left(x^{n-1}\right)$ term in the lowering operator we obtain

$$
n \boldsymbol{I}+\boldsymbol{a}_{n, n-1} \boldsymbol{A}-\boldsymbol{A} \boldsymbol{a}_{n, n-1}=4\left(\boldsymbol{\beta}_{n} \boldsymbol{\beta}_{n-1}+\boldsymbol{\beta}_{n} \boldsymbol{\alpha}_{n-1}^{2}+\boldsymbol{\alpha}_{n} \boldsymbol{\beta}_{n} \boldsymbol{\alpha}_{n-1}+\boldsymbol{\beta}_{n+1} \boldsymbol{\beta}_{n}+\boldsymbol{\beta}_{n}^{2}+\boldsymbol{\alpha}_{n}^{2} \boldsymbol{\beta}_{n}\right) .
$$

An expression of the second-order differential equation can be given using Proposition 3.1, but in this case it is necessary to compute the inverse of $\mathcal{A}_{n}(x ; \boldsymbol{G})$, which is a matrix polynomial of degree 2 with a rational function determinant in general. 


\subsection{2 $U^{\prime}(x) U^{-1}(x)=2 B x$}

The weight matrix (4.1) is given by

$$
\boldsymbol{W}(x)=e^{-x^{4}} e^{\boldsymbol{B} x^{2}} e^{\boldsymbol{B}^{*} x^{2}}, \quad \boldsymbol{B} \in \mathbb{C}^{N \times N}, \quad x \in \mathbb{R},
$$

we have $\boldsymbol{T}(x)=e^{-x^{4} / 2} e^{\boldsymbol{B} x^{2}}$ and

$$
\boldsymbol{G}(x)=-2 x^{3} \boldsymbol{I}+2 \boldsymbol{B} x
$$

Again, this weight matrix is an even matrix function, and we conclude as above that $\boldsymbol{a}_{n, n-1}=\mathbf{0}$ and $\boldsymbol{\alpha}_{n}=\mathbf{0}$. The coefficients (3.15) and (3.16) are given by (using the same approach as before)

$$
\mathcal{A}_{n}(x ; \boldsymbol{G})=4\left(x^{2} \boldsymbol{I}+\boldsymbol{\beta}_{n}^{*}+\boldsymbol{\beta}_{n+1}^{*}\right)-2\left(\boldsymbol{B}^{*}+\boldsymbol{\gamma}_{n} \boldsymbol{B} \boldsymbol{\gamma}_{n}^{-1}\right),
$$

and

$$
\mathcal{B}_{n}(x ; \boldsymbol{G})=2\left(x^{3} \boldsymbol{I}+\left(2 \boldsymbol{\beta}_{n}-\boldsymbol{B}\right) x\right) .
$$

Again, there is only one compatibility condition (3.23):

$$
\begin{aligned}
\boldsymbol{I}= & 4\left(\left(\boldsymbol{\beta}_{n+1}+\boldsymbol{\beta}_{n+2}\right) \boldsymbol{\beta}_{n+1}-\boldsymbol{\beta}_{n}\left(\boldsymbol{\beta}_{n}+\boldsymbol{\beta}_{n-1}\right)\right) \\
& -2\left(\boldsymbol{B} \boldsymbol{\beta}_{n+1}-\boldsymbol{\beta}_{n} \boldsymbol{B}+\boldsymbol{\gamma}_{n+1}^{-1} \boldsymbol{B}^{*} \boldsymbol{\gamma}_{n}-\boldsymbol{\gamma}_{n}^{-1} \boldsymbol{B}^{*} \boldsymbol{\gamma}_{n-1}\right)
\end{aligned}
$$

with the lowering and raising operators

$$
\begin{aligned}
\widehat{\boldsymbol{P}}_{n}^{\prime}(x) & +2 x\left(\widehat{\boldsymbol{P}}_{n}(x) \boldsymbol{B}-\boldsymbol{B} \widehat{\boldsymbol{P}}_{n}(x)\right)+4 x \boldsymbol{\beta}_{n} \widehat{\boldsymbol{P}}_{n}(x) \\
= & \left(4\left(x^{2} \boldsymbol{I}+\boldsymbol{\beta}_{n+1}+\boldsymbol{\beta}_{n}\right)-2\left(\boldsymbol{B}+\boldsymbol{\gamma}_{n}^{-1} \boldsymbol{B}^{*} \boldsymbol{\gamma}_{n}\right)\right) \boldsymbol{\beta}_{n} \widehat{\boldsymbol{P}}_{n-1}(x)
\end{aligned}
$$

and

$$
\begin{aligned}
\widehat{\boldsymbol{P}}_{n}^{\prime}(x) & +2 x\left(\widehat{\boldsymbol{P}}_{n}(x) \boldsymbol{B}-\boldsymbol{B} \widehat{\boldsymbol{P}}_{n}(x)\right)=\left(4 x^{3} \boldsymbol{I}+2\left(2 \boldsymbol{\beta}_{n+1}-\boldsymbol{B}-\boldsymbol{\gamma}_{n}^{-1} \boldsymbol{B}^{*} \boldsymbol{\gamma}_{n}\right) x\right) \widehat{\boldsymbol{P}}_{n}(x) \\
+ & \left(-4\left(x^{2} \boldsymbol{I}+\boldsymbol{\beta}_{n+1}+\boldsymbol{\beta}_{n}\right)+2\left(\boldsymbol{B}+\boldsymbol{\gamma}_{n}^{-1} \boldsymbol{B}^{*} \boldsymbol{\gamma}_{n}\right)\right) \widehat{\boldsymbol{P}}_{n+1}(x),
\end{aligned}
$$

respectively.

Finally, considering the $\mathcal{O}\left(x^{n-2}\right)$ term in the lowering operator we obtain

$$
n \boldsymbol{I}+2\left(\boldsymbol{a}_{n, n-2} \boldsymbol{B}-\boldsymbol{B} \boldsymbol{a}_{n, n-2}\right)=4\left(\boldsymbol{\beta}_{n} \boldsymbol{\beta}_{n-1}+\boldsymbol{\beta}_{n}^{2}+\boldsymbol{\beta}_{n+1} \boldsymbol{\beta}_{n}\right)-2\left(\boldsymbol{B}+\boldsymbol{\gamma}_{n}^{-1} \boldsymbol{B}^{*} \boldsymbol{\gamma}_{n}\right) \boldsymbol{\beta}_{n}
$$

This equation may be regarded of as a matrix version of a discrete Painlevé equation. Observe that in the scalar situation $(\boldsymbol{B}=\mathbf{0})$, the equation (4.18) reduces to the well-known string equations

$$
n=4 \beta_{n}\left(\beta_{n+1}+\beta_{n}+\beta_{n-1}\right)
$$

studied in the context of discrete Painlevé equations and orthogonal polynomials of Freud type in [52] (see formula (19) therein). 


\section{Final remarks}

This paper extends to the matrix case the methodology of derivation of differential relations for MOPRL using a RH approach. We obtain explicit formulas for the ladder operators of very general weight matrices factorized in the form $\boldsymbol{W}=\boldsymbol{T} \boldsymbol{T}^{*}$. We have also shown that in the matrix case there is an extra freedom absent in the scalar situation, which allows us to obtain a family of ladder operators, some of them of the 0-th order, which does not occur in the scalar case. Furthermore, by combining appropriately the family of ladder operators we get a family of second order differential equations satisfied by the MOPRL, some of them of the 0-th or first order (under some invertibility assumptions). This yields some new results even in the particular cases studied before in the literature.

In order to keep the size of this paper reasonable, we have restricted our attention to the weights supported on the whole real line. When $\operatorname{supp}(\boldsymbol{W})=[0,+\infty)$ or $\operatorname{supp}(\boldsymbol{W})=[-1,1]$, we can follow the ideas exposed in Section 3.1, except that now we have to assume that either $z \boldsymbol{G}(z)$ (for the case of the semi-axis) or $\left(1-z^{2}\right) \boldsymbol{G}(z)$ (for the case of the finite interval) are matrix polynomials. For the discussion of these situations in the scalar case, where the differential equations for the Laguerre and Jacobi polynomials are obtained, the interested reader is referred to [40, Chapter 22].

\section{Acknowledgements}

The authors are grateful for the excellent job of the referees, whose suggestions and remarks improved the final text.

The research of the first author was supported in part by the Applied Math. Sciences subprogram of the Office of Energy Research, USDOE, under Contract DE-AC03-76SF00098.

The work of the second author is partially supported by the research project MTM200912740-C03-02 from the Ministry of Science and Innovation of Spain and the European Regional Development Fund (ERDF), by Junta de Andalucía grant FQM-262, by K.U. Leuven research grant OT/04/21, and by Subprograma de estancias de movilidad posdoctoral en el extranjero, MICINN, ref. -2008-0207.

The third author is supported in part by Junta de Andalucía grant FQM-229, and by the research project MTM2008-06689-C02-01 from the Ministry of Science and Innovation of Spain and the European Regional Development Fund (ERDF).

Both the second and the third authors gratefully acknowledge also the support of Junta de Andalucía via the Excellence Research Grant P09-FQM-4643.

\section{References}

[1] Álvarez-Fernández C., Fidalgo U., Mañas M., The multicomponent 2D Toda hierarchy: generalized matrix orthogonal polynomials, multiple orthogonal polynomials and Riemann-Hilbert problems, Inverse Problems 26 (2010), 055009, 15 pages, arXiv:0911.0941.

[2] Berezans'kiǔ Ju.M., Expansions in eigenfunctions of selfadjoint operators, Translations of Mathematical Monographs, Vol. 17, American Mathematical Society, Providence, R.I., 1968.

[3] Borrego J., Castro M.M., Durán A.J., Orthogonal matrix polynomials satisfying differential equations with recurrence coefficients having non-scalar limits, arXiv:1102.1578.

[4] Cassatella-Contra G.A., Mañas M., Riemann-Hilbert problems, matrix orthogonal polynomials and discrete matrix equations with singularity confinement, Stud. Appl. Math., to appear, arXiv:1106.0036.

[5] Castro M.M., Grünbaum F.A., Orthogonal matrix polynomials satisfying first order differential equations: a collection of instructive examples, J. Nonlinear Math. Phys. 12 (2005), suppl. 2, 63-76.

[6] Castro M.M., Grünbaum F.A., The non-commutative bispectral problem for operators of order one, Constr. Approx. 27 (2008), 329-347. 
[7] Chen Y., Ismail M.E.H., Ladder operators and differential equations for orthogonal polynomials, J. Phys. A: Math. Gen. 30 (1997), 7817-7829.

[8] Daems E., Kuijlaars A.B.J., Multiple orthogonal polynomials of mixed type and non-intersecting Brownian motions, J. Approx. Theory 146 (2007), 91-114, math.CA/0511470.

[9] Daems E., Kuijlaars A.B.J., Veys W., Asymptotics of non-intersecting Brownian motions and a $4 \times 4$ Riemann-Hilbert problem, J. Approx. Theory 153 (2008), 225-256, math.CV/0701923.

[10] Dai D., Kuijlaars A.B.J., Painlevé IV asymptotics for orthogonal polynomials with respect to a modified Laguerre weight, Stud. Appl. Math. 122 (2009), 29-83, arXiv:0804.2564.

[11] Damanik D., Pushnitski A., Simon B., The analytic theory of matrix orthogonal polynomials, Surv. Approx. Theory 4 (2008), 1-85, arXiv:0711.2703.

[12] Deift P.A., Orthogonal polynomials and random matrices: a Riemann-Hilbert approach, Courant Lecture Notes in Mathematics, Vol. 3, New York University Courant Institute of Mathematical Sciences, New York, 1999.

[13] Deift P.A., Riemann-Hilbert methods in the theory of orthogonal polynomials, in Spectral Theory and Mathematical Physics: a Festschrift in Honor of Barry Simon's 60th Birthday, Proc. Sympos. Pure Math., Vol. 76, Amer. Math. Soc., Providence, RI, 2007, 715-740, math.CA/0603309.

[14] Deift P.A., Gioev D., Random matrix theory: invariant ensembles and universality, Courant Lecture Notes in Mathematics, Vol. 18, Courant Institute of Mathematical Sciences, New York, 2009.

[15] Deift P.A., Kamvissis S., Kriecherbauer Th., Zhou X., The Toda rarefaction problem, Comm. Pure Appl. Math. 49 (1996), 35-83.

[16] Deift P.A., Zhou X., A steepest descent method for oscillatory Riemann-Hilbert problems. Asymptotics for the MKdV equation, Ann. of Math. (2) 137 (1993), 295-368, math.AP/9201261.

[17] Deift P.A., Zhou X., Long-time asymptotics for solutions of the NLS equation with initial data in a weighted Sobolev space, Comm. Pure Appl. Math. 56 (2003), 1029-1077, math.AP/0206222.

[18] Delvaux S., Average characteristic polynomials for multiple orthogonal polynomial ensembles, J. Approx. Theory 162 (2010), 1033-1067, arXiv:0907.0156.

[19] Delvaux S., Kuijlaars A.B.J., A phase transition for nonintersecting Brownian motions, and the Painlevé equation, Int. Math. Res. Not. 2009 (2009), no. 19, 3639-3725, arXiv:0809.1000.

[20] Durán A.J., Markov's theorem for orthogonal matrix polynomials, Canad. J. Math. 48 (1996), $1180-1195$.

[21] Durán A.J., Matrix inner product having a matrix symmetric second order differential operator, Rocky Mountain J. Math. 27 (1997), 585-600.

[22] Durán A.J., Generating orthogonal matrix polynomials satisfying second order differential equations from a trio of triangular matrices, J. Approx. Theory 161 (2009), 88-113.

[23] Durán A.J., Grünbaum F.A., Orthogonal matrix polynomials satisfying second-order differential equations, Int. Math. Res. Not. 2004 (2004), no. 10, 461-484.

[24] Durán A.J., Grünbaum F.A., A characterization for a class of weight matrices with orthogonal matrix polynomials satisfying second-order differential equations, Int. Math. Res. Not. 2005 (2005), no. 23, 13711390.

[25] Durán A.J., Ismail M.E.H., Differential coefficients of orthogonal matrix polynomials, J. Comput. Appl. Math. 190 (2006), 424-436.

[26] Durán A.J., Van Assche W., Orthogonal matrix polynomials and higher-order recurrence relations, Linear Algebra Appl. 219 (1995), 261-280, math.CA/9310220.

[27] Flaschka H., The Toda lattice. II. Existence of integrals, Phys. Rev. B 9 (1974), 1924-1925.

[28] Fokas A.S., Its A.R., Kitaev A.V., The isomonodromy approach to matrix models in 2D quantum gravity, Comm. Math. Phys. 147 (1992), 395-430.

[29] Grünbaum F.A., Matrix valued Jacobi polynomials, Bull. Sci. Math. 127 (2003), 207-214.

[30] Grünbaum F.A., Random walks and orthogonal polynomials: some challenges, in Probability, Geometry and Integrable Systems, Math. Sci. Res. Inst. Publ., Vol. 55, Cambridge Univ. Press, Cambridge, 2008, 241-260.

[31] Grünbaum F.A., Block tridiagonal matrices and a beefed-up version of the Ehrenfest urn model, in Modern Analysis and Applications. The Mark Krein Centenary Conference, Vol. 1, Operator Theory and Related Topics, Oper. Theory Adv. Appl., Vol. 100, Birkhäuser Verlag, Basel, 2009, 267-277. 
[32] Grünbaum F.A., A spectral weight matrix for a discrete version of Walsh's spider, in Topics in Operator Theory, Vol. 1, Operators, Matrices and Analytic Functions, Oper. Theory Adv. Appl., Vol. 202, Birkhäuser Verlag, Basel, 2010, 253-264.

[33] Grünbaum F.A., de la Iglesia M.D., Matrix valued orthogonal polynomials arising from group representation theory and a family of quasi-birth-and-death processes, SIAM J. Matrix Anal. Appl. 30 (2008), 741-761.

[34] Grünbaum F.A., Pacharoni I., Tirao J., Matrix valued spherical functions associated to the complex projective plane, J. Funct. Anal. 188 (2002), 350-441, math.RT/0108042.

[35] Grünbaum F.A., Pacharoni I., Tirao J., Matrix valued orthogonal polynomials of the Jacobi type, Indag. Math. (N.S.) 14 (2003), 353-366.

[36] Grünbaum F.A., Pacharoni I., Tirao J., An invitation to matrix-valued spherical functions: linearization of products in the case of complex projective space $P_{2}(\mathbb{C})$, in Modern Signal Processing, Math. Sci. Res. Inst. Publ., Vol. 46, Cambridge Univ. Press, Cambridge, 2004, 147-160, math.RT/0202304.

[37] Grünbaum F.A., Pacharoni I., Tirao J., Two stochastic models of a random walk in the $U(n)$ spherical duals of $U(n+1)$, arXiv:1010.0720.

[38] Grünbaum F.A., Tirao J., The algebra of differential operators associate to a weight matrix, Integr. Equ. Oper. Theory 58 (2007), 449-475.

[39] Hille E., Ordinary differential equations in the complex domain, Pure and Applied Mathematics, WileyInterscience, New York, 1976.

[40] Ismail M.E.H., Classical and quantum orthogonal polynomials in one variable, Encyclopedia of Mathematics and its Applications, Vol. 98, Cambridge University Press, Cambridge, 2005.

[41] Its A.R., Kuijlaars A.B.J., Östensson J., Asymptotics for a special solution of the thirty fourth Painlevé equation, Nonlinearity 22 (2009), 1523-1558, arXiv:0811.3847.

[42] Kreĭn M.G., Infinite J-matrices and a matrix-moment problem, Doklady Akad. Nauk SSSR (N.S.) 69 (1949), 125-128 (in Russian).

[43] Kreı̆n M.G., Fundamental aspects of the representation theory of Hermitian operators with deficiency in$\operatorname{dex}(m, m)$, Am. Math. Soc. Translat. Ser. 297 (1971), 75-143.

[44] Kuijlaars A.B.J., Martínez-Finkelshtein A., Wielonsky F., Non-intersecting squared Bessel paths and multiple orthogonal polynomials for modified Bessel weights, Comm. Math. Phys. 286 (2009), 217-275, arXiv:1011.1278.

[45] Kuijlaars A.B.J., Multiple orthogonal polynomial ensembles, in Recent Trends in Orthogonal Polynomials and Approximation Theory, Contemp. Math., Vol. 507, Amer. Math. Soc., Providence, RI, 2010, 155-176, arXiv:0902.1058.

[46] McLaughlin K.T.-R., Vartanian A.H., Zhou X., Asymptotics of Laurent polynomials of even degree orthogonal with respect to varying exponential weights, IMRP Int. Math. Res. Pap. 2006 (2006), Art. ID 62815, 216 pages, math.CA/0601306.

[47] McLaughlin K.T.-R., Vartanian A.H., Zhou X., Asymptotics of Laurent polynomials of odd degree orthogonal with respect to varying exponential weights, Constr. Approx. 27 (2008), 149-202, math.CA/0601595.

[48] Miranian L., Matrix-valued orthogonal polynomials on the real line: some extensions of the classical theory, J. Phys. A: Math. Gen. 38 (2005), 5731-5749.

[49] Sibuya Y., Linear differential equations in the complex domain: problems of analytic continuation, Translations of Mathematical Monographs, Vol. 82, American Mathematical Society, Providence, RI, 1990.

[50] Simon B., Orthogonal polynomials on the unit circle I and II, AMS Colloquium Publications, Vol. 54, American Mathematical Society, Providence, RI, 2005.

[51] Simon B., Szegö's theorem and its descendants: spectral theory for $L^{2}$ perturbations of orthogonal polynomials, Princeton University Press, 2010.

[52] Van Assche W., Discrete Painlevé equations for recurrence coefficients of orthogonal polynomials, in Difference Equations, Special Functions and Orthogonal Polynomials, World Sci. Publ., Hackensack, NJ, 2007, 687-725, math.CA/0512358. 